\title{
Integrated Energy and Flux Balance Based Multiobjective Framework for Large-Scale Metabolic Networks
}

\author{
Deepak Nagrath, ${ }^{1,2}$ Marco Avila-Elchiver, ${ }^{1,2}$ Francois Berthiaume, ${ }^{1,2}$ Arno W. Tilles, ${ }^{1,2}$ Achille \\ Messac, ${ }^{3}$ and Martin L. Yarmush ${ }^{1,2}$ \\ ${ }^{1}$ Center for Engineering in Medicine/Surgical Services, Massachusetts General Hospital, Harvard Medical School, Boston, MA \\ 02114, USA; ${ }^{2}$ Shriners Burns Hospital, 51 Blossom Street, Boston, MA 02114, USA; and ${ }^{3}$ Department of Mechanical \\ Engineering, Rensselaer Polytechnic Institute, Troy, NY, USA
}

(Received 22 September 2006; accepted 13 February 2007; published online 29 March 2007)

\begin{abstract}
Flux balance analysis (FBA) provides a framework for the estimation of intracellular fluxes and energy balance analysis (EBA) ensures the thermodynamic feasibility of the computed optimal fluxes. Previously, these techniques have been used to obtain optimal fluxes that maximize a single objective. Because mammalian systems perform various functions, a multi-objective approach is needed when seeking optimal flux distributions in such systems. For example, hepatocytes perform several metabolic functions at various levels depending on environmental conditions; furthermore, there is a potential benefit to enhance some of these functions for applications such as bioartificial liver (BAL) support devices. Herein we developed a multi-objective optimization approach that couples the normalized Normal Constraint (NC) with both FBA and EBA to obtain multi-objective Pareto-optimal solutions. We investigated the Pareto frontiers in gluconeogenic and glycolytic hepatocytes for various combinations of liver-specific objectives (albumin synthesis, glutathione synthesis, NADPH synthesis, ATP generation, and urea secretion). Next, we evaluated the impact of experimental flux measurements on the Pareto frontiers. We found that measurements induce dramatic changes in Pareto frontiers and further constrain the network fluxes. This multi-objective optimality analysis may help explain certain features of the metabolic control of hepatocytes, which is relevant to the response to hepatocytes and liver to various physiological stimuli and disease states.
\end{abstract}

Keywords-Metabolic networks, Energy balance analysis, Flux balance analysis, Multi-objective optimization, Pareto optimality, Hepatocytes, Bioartificial liver.

\section{INTRODUCTION}

The quantification of intracellular metabolic fluxes is widely used for investigation of the metabolism in mioorganisms $s^{9,10,17-21}$ and mammalian sys-

Address correspondence to Martin L. Yarmush, Shriners Burns Hospital, 51 Blossom Street, Boston, MA 02114, USA. Electronic mail: ireis@sbi.org tems. ${ }^{1,5-8,11-14,24,25}$ Flux balance analysis (FBA) uses stoichiometric and mass balance constraints to compute the intracellular fluxes. Recently, energy balance analysis (EBA) was developed to ensure the thermodynamic feasibility of the computed fluxes. ${ }^{2-4,23}$ EBA imposes the thermodynamic constraints on reaction fluxes both explicitly and implicitly. Essentially, the reaction potentials are computed based on the chemical potentials and then these are used to obtain thermodynamic constraints that are based on the first and second laws of thermodynamics. Thermodynamic constraints further reduce the feasible solution space based on stoichiometric constraints alone. Available measurements, which bring in environmental constraints such as certain cell culture conditions, medium supplements, induced stress and extracellular matrices, typically limit the feasible solution space even further. If a sufficient number of measurements is available, the analysis may yield a unique solution.

Since mammalian systems perform an array of metabolic functions (protein secretion, detoxification, energy production), several different objectives need to be taken into account simultaneously when seeking optimal fluxes. Typically, several objectives compete against each other; therefore, only "Pareto-optimal" solutions can be achieved. A solution is said to be Pareto-optimal if there are no other solutions that can better satisfy all of the objectives simultaneously. ${ }^{16}$ Specifically, a Pareto solution is one where any improvement in one objective necessitates the worsening of at least one other objective. Non-Paretooptimal solutions are sub-optimal and their performance is inferior to systems operating and designed based on Pareto optimality of objectives. The class of Generate First-Choose Later (GCFL) multi-objective optimization approaches entails first generating a representative set of Pareto solutions, and then choosing the most suitable and appropriate solution 
within this set. The Normal Constraint (NC) method, ${ }^{15}$ unlike other popular methods such as the Normal Boundary Intersection (NBI) method, can generate complete Pareto frontiers for multi-objective problems from the full range of Pareto bi-objective solutions. The NC method essentially generates an even distribution of Pareto points throughout the complete Pareto frontier; and it is guaranteed to yield any Pareto point in the feasible design space. Further, it is insensitive to objective function scaling, and is valid for any arbitrary number of design objectives. Figure 1a shows the reduced feasible space because of various constraints (stoichiometric, environmental, and thermodynamic) changes the Pareto surface. As seen in the figure, the decreased Pareto hypersurface area ultimately results in the decreased Pareto frontiers when the hypersurface is projected in two-dimensions.

In the current work, we develop a Normalized Constraint Energy and Flux Balance Analysis (NCEFBA) based multi-objective framework for characterizing the intermediary metabolism of largescale metabolic networks. The implementation is general and could be easily modified for other metabolic networks but here it is presented in the context of hepatic metabolism. In the context of a bioartificial liver (BAL) device, this multi-objective optimal flux analysis

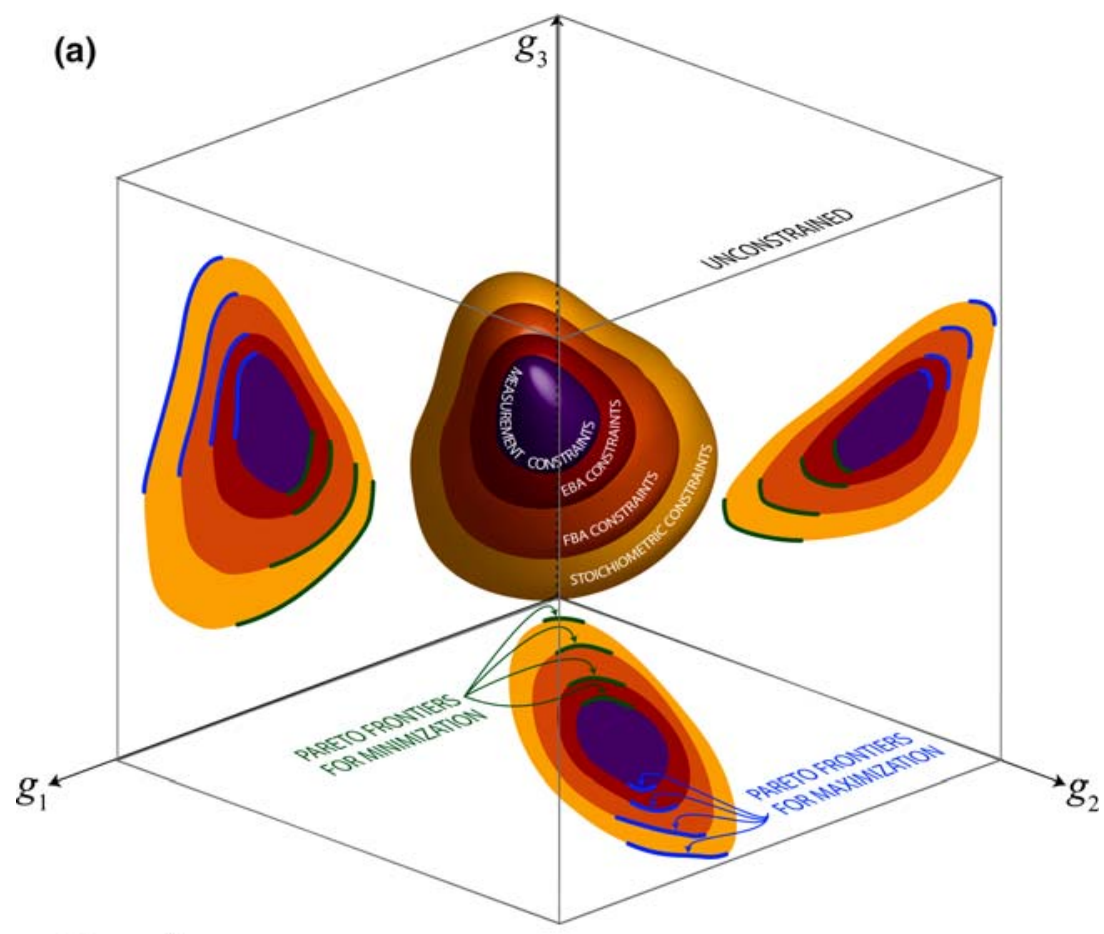

(b)

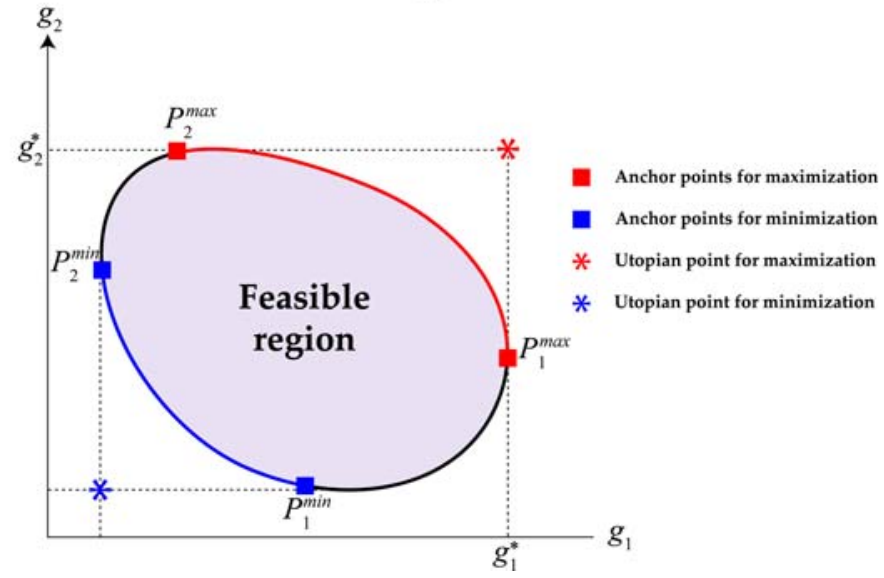

FIGURE 1. (a) Feasible space reduction due to of the imposed stoichiometric, flux balance, energy balance, and measurement constraints. The Pareto surface of the feasible space is projected onto the $g_{1} g_{2}, g_{2} g_{3}$, and $g_{1} g_{3}$ planes and their corresponding Pareto frontiers are shown. The mutually orthogonal axes $g_{1}, g_{2}$, and $g_{3}$, represent the individual design objectives. (b) Pareto frontiers and Pareto-optimal solutions shown are for bi-objective maximization and minimization problems. 
could play an important role in: (a) understanding the underlying mechanisms of perturbing a sub-optimal hepatic cellular system towards an optimal state, (b) optimizing hepatocyte functions in an extracorporeal BAL device, (c) studying the intracellular activity of liver under various physiological and disease states, and (d) the preconditioning and preservation of donor livers. The presented multi-objective optimization platform NCEFBA couples the normalized NC method with both FBA and EBA to obtain multiobjective Pareto-optimal solutions.

Here the NCEFBA method was implemented to investigate Pareto-optimal solutions for the hepatic metabolic network under both gluconeogenic and glycolytic conditions. We analyzed various combinations of liver-specific objectives (albumin synthesis, glutathione synthesis, NADPH synthesis, ATP generation, and urea secretion). Next, the sensitivity to available measurements of these Pareto frontiers and changes in objective inter-optimality is presented. Noticeably, measurements induced dramatic changes in Pareto frontiers and further constrained the network fluxes.

\section{THEORY}

\section{Metabolic Flux Analysis}

The stoichiometric coefficients of the metabolic reactions are collected into a matrix $S$, where each element $s_{i j}$ is the coefficient of metabolite $i$ in reaction $j$. $S$ has dimensions of $M \times N$, where $M$ is the number of metabolites and $N$ is the number of reactions. In matrix form the mass balance is written as:

$$
\frac{d x}{d t}=S J,
$$

where each element $x_{i}$ of $x$ is the intracellular concentration of metabolite $i$ and element $J_{i}$ of $J$ is the net rate of conversion in reaction $j$. External metabolite fluxes are generally measured (e.g., uptake of glucose, lactate, amino acids). Because of the very high turnover of the intracellular pools of most intracellular metabolites, the time scale of the intracellular metabolic reactions is short compared to other cellular reactions. Hence, the pseudo steady state assumption is generally applied to the metabolite mass balances and thus

\section{Stoichiometric Equality Constraints for Unmeasured Fluxes}

$$
S J=0 \text { (Mass Balance Constraint) }
$$

Stoichiometric Equality Constraints for Measured Fluxes

$$
S_{u} J_{u}=-S_{m} J_{m} \text { (Mass Balance Constraint) }
$$

where $J_{m}$ and $J_{u}$ indicates measured and unmeasured fluxes, respectively, and $S_{m}$ and $S_{u}$ contain the stoichiometric coefficients of measured and unknown reactions, respectively.

A previously described hepatic metabolic network $^{5-8}$ includes all of the major intracellular pathways that account for the majority of central carbon and nitrogen metabolism found in hepatocytes, namely the tricarboxylic acid (TCA) and urea cycles, the gluconeogenic and glycolytic pathways, the pentose phosphate shunt, pathways of entry, transamination, and deamination of amino acids, protein synthesis, and the major components of lipid metabolism, including triglyceride synthesis and breakdown and $\beta$-oxidation of fatty acids, in addition to amino acid synthesis and apolipoprotein degradation. The current hepatic metabolic network model (Table 1) includes a few additional reactions, namely those of the 3phosphoglycerate cycle as it is involved in glycerol production and glutathione synthesis reaction, which results in a total of 81 reactions (as compared to 76 reactions in the previous model) and 47 metabolites (Table 2). Glutathione is an important mediator in detoxification reactions of hepatocytes. The model assumes pseudosteady-state with no metabolic futile cycles. These assumptions are discussed in detail elsewhere. ${ }^{5-8}$

\section{Energy Balance Analysis}

Energy Balance Analysis imposes constraints based on law of thermodynamics on the cellular reaction networks. ${ }^{2,3}$ For any reaction set, if stoichiometry is represented by matrix $S, \mu$ denotes an $M$-dimensional vector of chemical potentials, $\Delta \mu$ denotes the $N$-dimensional vector of reaction potentials, then these potentials can be computed as $\Delta \mu=S^{T} \mu$. The null space matrix of $S$ (for $r$ linearly independent rows, with $r \leq N$ ) is denoted by $K$ and forms a basis for the null space of $S$, so that $S K=0$. The product of the null space $K$ of the stoichiometric $S$ with the chemical potential difference gives the energy balance equation as $K \Delta \mu=0$. This balances the global potential energy of the network. The first law of thermodynamics necessitates energy conservation, which then leads to an equality constraint as

$$
\begin{aligned}
K^{T} \Delta \mu= & K^{T} S^{T} \mu=0 \\
& \text { (First Law of Thermodynamics-based } \\
& \text { Energy Equality Constraint) }
\end{aligned}
$$

This constraint requires that the sum of reaction potentials around any cycle of reactions equals zero, which is similar to Kirchoff's voltage or loop law of electrical circuit theory, and is known as the energy balance constraint of EBA. ${ }^{2-4}$ The second law of 
TABLE 1. Hepatic stoichiometric reactions.

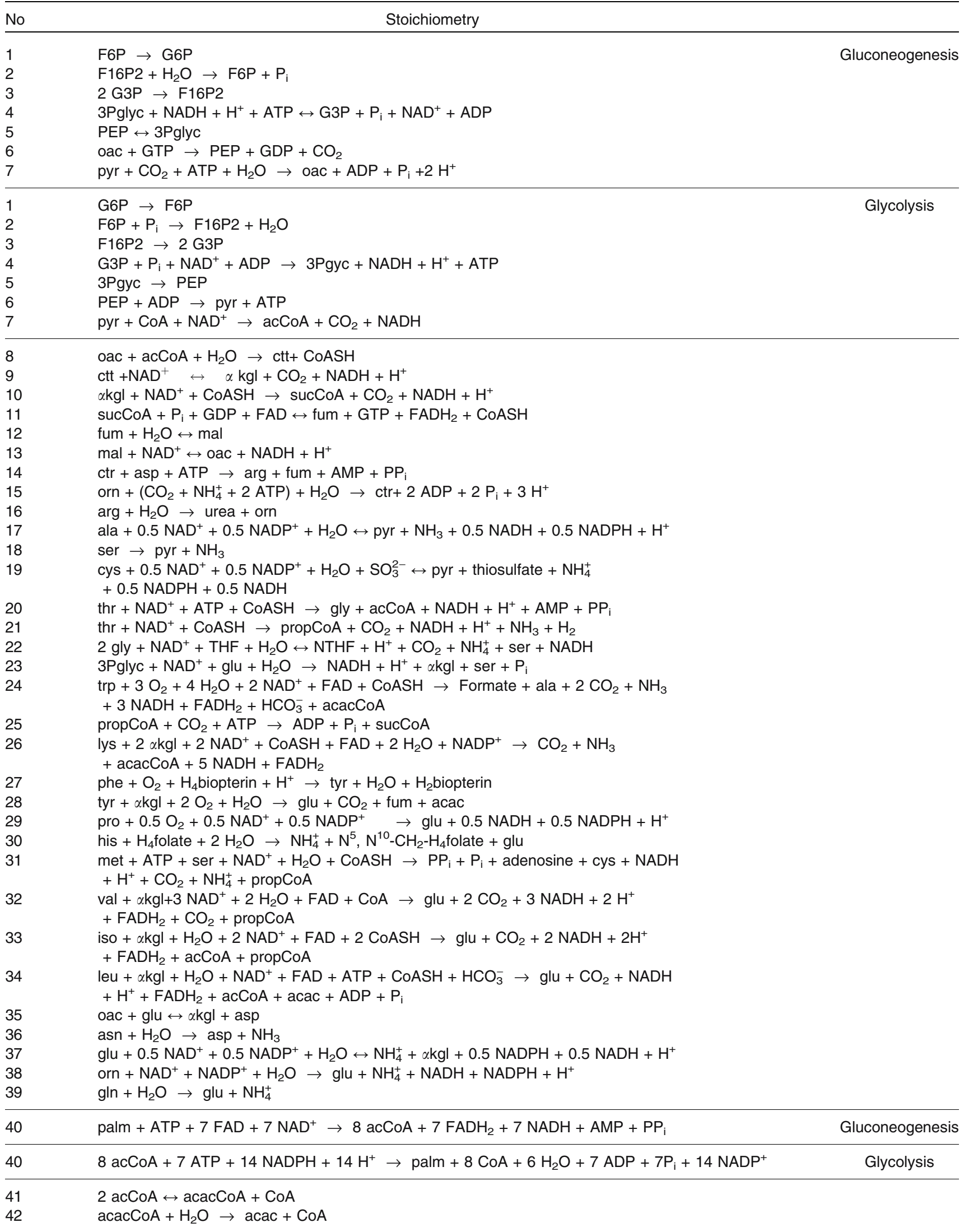


TABLE 1. Continued.

\begin{tabular}{|c|c|}
\hline No & Stoichiometry \\
\hline 43 & $\mathrm{NADH}+\mathrm{H}^{+}+0.5 \mathrm{O}_{2}+3 \mathrm{ADP} \rightarrow \mathrm{NAD}^{+}+\mathrm{H}_{2} \mathrm{O}+3 \mathrm{ATP}$ \\
\hline 44 & $\mathrm{FADH}_{2}+0.5 \mathrm{O}_{2}+2 \mathrm{ADP} \rightarrow \mathrm{FAD}+\mathrm{H}_{2} \mathrm{O}+2 \mathrm{ATP}$ \\
\hline 45 & $\mathrm{gol}+\mathrm{NAD}^{+}+\mathrm{ATP} \leftrightarrow \mathrm{G} 3 \mathrm{P}+\mathrm{NADH}+\mathrm{H}^{+}+\mathrm{ADP}+\mathrm{P}_{\mathrm{i}}$ \\
\hline 46 & $\mathrm{G} 6 \mathrm{P}+12 \mathrm{NADP}^{+}+7 \mathrm{H}_{2} \mathrm{O} \rightarrow 6 \mathrm{CO}_{2}+12 \mathrm{NADPH}+12 \mathrm{H}^{+}+\mathrm{P}_{\mathrm{i}}$ \\
\hline 47 & $\begin{array}{l}24 \text { arg }+32 \text { asp }+61 \text { ala }+24 \text { ser }+35 \text { cys }+57 \text { glu }+17 \text { gly }+21 \text { tyr }+33 \text { thr }+53 \text { lys } \\
+26 \text { phe }+25 \text { gln }+30 \text { pro }+15 \text { his }+6 \text { met }+20 \text { asn }+ \text { trp }+35 \text { val }+13 \text { iso }+56 \text { leu } \\
+2332 \text { ATP } \rightarrow \text { albumin }+2332 \text { ADP }+2332 \mathrm{P}_{\mathrm{i}}\end{array}$ \\
\hline 48 & glu +2 ATP + cys + gly + NADPH $\rightarrow \mathrm{GSH}+2 \mathrm{ADP}+2 \mathrm{P}_{\mathrm{i}}+\mathrm{NADP}^{+}+\mathrm{H}^{+}$ \\
\hline 49 & $\mathrm{gol}+3 \mathrm{acCoA}+\mathrm{H}_{2} \mathrm{O}+\mathrm{ATP} \rightarrow 3 \mathrm{CoASH}+\mathrm{P}_{\mathrm{i}}+\mathrm{TG}+\mathrm{ADP}+\mathrm{P}_{\mathrm{i}}$ \\
\hline 50 & lactate $+\mathrm{NAD}^{+} \leftrightarrow \mathrm{pyr}+\mathrm{NADH}+\mathrm{H}^{+}$ \\
\hline 51 & acac $+\mathrm{NADH}+\mathrm{H}^{+} \leftrightarrow \beta$-OH-butyrate $+\mathrm{NAD}^{+}$ \\
\hline 52 & $\mathrm{TG}+3 \mathrm{H}_{2} \mathrm{O} \rightarrow \mathrm{gol}+3 \mathrm{palm}+3 \mathrm{H}^{+}$ \\
\hline 53 & G6P release \\
\hline 54 & gol uptake \\
\hline 55 & palm release \\
\hline 56 & cholesterol ester $+\mathrm{H}_{2} \mathrm{O} \rightarrow$ cholesterol + palm \\
\hline 57 & TG stored \\
\hline 58 & trp uptake \\
\hline 59 & $\mathrm{O}_{2}$ uptake \\
\hline 60 & pro uptake \\
\hline 61 & glu secretion \\
\hline 62 & asn uptake \\
\hline 63 & orn secretion \\
\hline 64 & arg uptake \\
\hline 65 & $\mathrm{NH}_{4}^{+}$uptake \\
\hline 66 & ala uptake \\
\hline 67 & ser uptake \\
\hline 68 & gly uptake \\
\hline 69 & asp uptake \\
\hline 70 & acac production \\
\hline 71 & thr uptake \\
\hline 72 & lys uptake \\
\hline 73 & phe uptake \\
\hline 74 & his uptake \\
\hline 75 & met uptake \\
\hline 76 & val uptake \\
\hline 77 & iso uptake \\
\hline 78 & leu uptake \\
\hline 79 & gln uptake \\
\hline 80 & cys uptake \\
\hline 81 & tyr uptake \\
\hline
\end{tabular}

thermodynamics takes the form of an inequality constraint for each flux as $-J_{i} \Delta \mu_{i} \geq 0$. However, this equation is written in terms of net fluxes. Beard et al. ${ }^{3}$ compute the net flux distribution through the reaction network by introducing the concept of reversibility of each reaction which entails defining the non-negative forward and reverse reaction fluxes, $J_{+}$and $J_{-}$ respectively, with $j$ th entries representing the one-way fluxes through the $j$ th reaction. The vector of net flux distribution through the reaction network can be then computed as $J=J_{+}-J_{-}$, which is then used to compute the $j$ th reaction potential as

$$
\Delta \mu^{j}=R T \ln \left(\frac{J_{-}^{j}}{J_{+}^{j}}\right)
$$

where $R$ is the ideal gas constant and $T$ is the temperature.

This relationship leads directly to the second law of thermodynamics, i.e.,

$$
-J^{j} \Delta \mu^{j}=-R T\left(J_{+}^{j}-J_{-}^{j}\right) \ln \left(\frac{J_{-}^{j}}{J_{+}^{j}}\right) \geq 0
$$

(Second Law of Thermodynamics-based

$$
\text { Energy Inequality Constraint) }
$$

which says that the system must dissipate heat, and entropy must increase as a result of the work being done on the system through the external fluxes. For equilibrium systems, this is an equality since for these systems $J^{j}=\Delta \mu^{j}=0$. 
TABLE 2. List of metabolites.

\begin{tabular}{|c|c|c|}
\hline No & Symbol & Metabolite \\
\hline 1 & G6P & Glucose-6-phosphate \\
\hline 2 & F6P & Fructose-6-phosphate \\
\hline 3 & F16P2 & Fructose-1,6-biphosphate \\
\hline 4 & G3P & Glyceraldehyde-3-phosphate \\
\hline 5 & PEP & Phosphoenolpyruvate \\
\hline 6 & oac & Oxaloacetate \\
\hline 7 & pyr & Pyruvate \\
\hline 8 & ctt & Citrate \\
\hline 9 & $\alpha \mathrm{kgl}$ & $\alpha$-Ketoglutarate \\
\hline 10 & sucCoA & Succinyl-CoA \\
\hline 11 & fum & Fumarate \\
\hline 12 & mal & Malate \\
\hline 13 & $\arg$ & Arginine \\
\hline 14 & orn & Ornithine \\
\hline 15 & ctr & Citrulline \\
\hline 16 & $\mathrm{NH}_{4}^{+}$ & Ammonium \\
\hline 17 & asp & Aspartate \\
\hline 18 & his & Histidine \\
\hline 19 & glu & Glutamate \\
\hline 20 & gln & Glutamine \\
\hline 21 & met & Methionine \\
\hline 22 & thr & Threonine \\
\hline 23 & val & Valine \\
\hline 24 & iso & Isoleucine \\
\hline 25 & phe & Phenylananine \\
\hline 26 & $\operatorname{trp}$ & Tryptophan \\
\hline 27 & lys & Lysine \\
\hline 28 & tyr & Tyrosine \\
\hline 29 & ala & Alanine \\
\hline 30 & asn & Asparagine \\
\hline 31 & pro & Proline \\
\hline 32 & cys & Cysteine \\
\hline 33 & ser & Serine \\
\hline 34 & gly & Glycine \\
\hline 35 & propCoA & Propionyl-CoA \\
\hline 36 & acCoA & Acetyl-CoA \\
\hline 37 & palm & Palmitate \\
\hline 38 & acacCoA & Acetoacetyl-Coa \\
\hline 39 & acac & Acetoacetate \\
\hline 40 & gol & Glycerol \\
\hline 41 & $\mathrm{NADH}$ & Nicotinamide adenine dinucleotide, reduced form \\
\hline 42 & NADPH & $\begin{array}{l}\text { Nicotinamide adenine dinucleotide phosphate, } \\
\text { reduced form }\end{array}$ \\
\hline 43 & $\mathrm{FADH}_{2}$ & Flavin adenine dinucleotide, reduced form \\
\hline 44 & $\mathrm{O}_{2}$ & Oxygen \\
\hline 45 & leu & Leucine \\
\hline 46 & 3Pglyc & 3-Phosphoglycerate \\
\hline 47 & TG & Triglyceride \\
\hline
\end{tabular}

The other inequality constraint is obtained for energy balance by ensuring that the total heat dissipation rate of the living system is always positive as indicated by

$$
\begin{aligned}
& h d r=-J^{T} \Delta \mu>0 \\
& \text { (Inequality Heat Dissipation Constraint) }
\end{aligned}
$$

Since, $h d r \rightarrow 0$ in the limit as $J_{-} / J_{+} \rightarrow 1$ component-wise while maintaining $J=J_{+}-J_{-}$so to prevent this physically unrealistic possibility, an additional inequality constraint

$$
\begin{aligned}
& (h d r)_{l b} \leq h d r \leq(h d r)_{u b} \\
& \quad \text { (Inequality Heat Dissipation Constraint) }
\end{aligned}
$$

is also imposed as part of energy balance analysis.

\section{Pareto Optimality}

Table 3 shows some of the definitions and mathematical formulation of the generic terms involved in multi-objective optimization. The mathematical representation of the generic multi-objective optimization problem is as follows.

Problem P1

$$
\min _{x}\left\{g_{1}(x), g_{2}(x), \ldots, g_{n}(x)\right\} \quad(n \geq 2)
$$

subject to:

$$
\begin{gathered}
f_{j}(x) \leq 0 \quad(1 \leq j \leq r) \\
h_{k}(x)=0 \quad(1 \leq k \leq s) \\
x_{l} \leq x_{i} \leq x_{u} \quad\left(1 \leq i \leq n_{x}\right)
\end{gathered}
$$

where the vector $x$ denotes the design variables and $g_{i}$ denotes the $i$ th objective function. Equations (9b)(9d) denote the inequality, equality and side constraints, respectively. Problem P1 does not yield a unique solution on its own, as it requires a preference or prioritization of objectives to obtain a single optimum solution. The NC method requires anchor points, $g_{i}{ }^{*}$, or optimum vertices to obtain the desired optimal solutions. The $i$ th anchor point (or end point) is obtained when the generic $i$ th objective is minimized independently. Figure $1 \mathrm{~b}$ presents a schematic of a Pareto set for a bi-objective problem. If the design metric $g_{1}$ alone is optimized (maximized), then the optimal value is $g_{1}^{*}\left(P_{1}\right)$. Similarly, if the design metric $g_{2}$ alone is optimized, then the optimal value is $g_{2}^{*}\left(P_{2}\right)$. Here $g_{1}^{*}$ and $g_{2}^{*}$ are the anchor values for design metrics $g_{1}$ and $g_{2}$, respectively. The ideal or Utopian solution $\left(g_{1}^{*}, g_{2}^{*}\right)$ obtained by the individual maximization of the objective functions is generally not a feasible solution of the multi-objective optimization problem. The arc joining points $P_{1}$ and $P_{2}$ is the Pareto frontier that represents the optimal tradeoff solutions. Generally, the desired solution can be chosen from the Pareto set; the line joining two anchor points in bi-objective cases, the utopia line, and the plane that comprises all anchor points in the multi-objective case, the utopia hyper plane. The anchor points are obtained by solving Problem PUi, defined as follows. 
TABLE 3. Definitions and mathematical formulations of some of the relevant multi-objective optimization keywords used in this paper.

\begin{tabular}{|c|c|c|}
\hline Terms & Mathematical formulation & Definition \\
\hline $\begin{array}{l}\text { Multi-objective } \\
\text { optimization }\end{array}$ & $\begin{array}{l}\text { Problem P1 } \\
\quad \min \left\{g_{1}(x), g_{2}(x), \ldots, g_{n}(x)\right\} \quad(n \geq 2) \\
\text { subjéct to } \\
\qquad f_{j}(x) \leq 0 \quad(1 \leq j \leq r) \\
\quad h_{k}(x)=0 \quad(1 \leq k \leq s) \\
\quad x_{I} \leq x_{i} \leq x_{u} \quad\left(1 \leq i \leq n_{x}\right) \\
\text { The vector } x \text { denotes the design variables } \\
\text { and } g_{i} \text { denotes the ith objective function }\end{array}$ & $\begin{array}{l}\text { A multi-objective optimization is a problem involving several } \\
\text { competing objectives and constraints. The solution of this } \\
\text { problem is considered the best solution that satisfies the } \\
\text { conflicting objectives }\end{array}$ \\
\hline
\end{tabular}

Pareto solution Solutions joining the anchor points and part of the feasible space
A Pareto solution is one where any improvement in one objective can only take place at the cost of another objective. A Pareto set is a set of Pareto-optimal solutions

Design parameters The vector $x$ denotes the design parameters

A design parameter is a parameter over which the designer has direct control. Other terms used in the literature for design parameters include decision variables, design variables or decision parameters

Design metric The variable $g(x)$ denotes the vector of design metrics

\begin{tabular}{ccc}
\hline Design constraint & $f_{j}(x) \leq 0$ & $(1 \leq j \leq r)$ \\
& $h_{k}(\boldsymbol{x})=0$ & $(1 \leq k \leq s)$ \\
& $x_{l} \leq x_{i} \leq x_{u}$ & $\left(1 \leq i \leq n_{x}\right)$
\end{tabular}

A design metric refers to an objective measure of a design attribute. Other commonly used terms are objective functions, design criterion, figure-of-merit, goal and performance metric

A design constraint indicates the lower or upper bounds in the design metrics or design parameters

The value obtained for a particular design objective if that design metric alone is optimized, given the bounds on the design parameters

$$
\begin{array}{cc}
\quad \min _{x}\left\{g_{i}(\boldsymbol{x})\right\} & (1 \leq i \leq n) \\
\text { subjêct to } & \\
f_{j}(\boldsymbol{x}) \leq 0 & (1 \leq j \leq r) \\
h_{k}(\boldsymbol{x})=0 & (1 \leq k \leq s) \\
\text { or } x_{I} \leq x_{i} \leq x_{u} & \left(1 \leq i \leq n_{x}\right) \\
g^{i *}=\left[g_{1}\left(x^{i *}\right)\right. & \left.g_{2}\left(x^{i *}\right) \ldots g_{n}\left(x^{i *}\right)\right]^{T} \\
\text { where } x^{i *}=\arg \left\{\min g_{i}(\boldsymbol{x})\right\}
\end{array}
$$

Nadir point

$$
\begin{aligned}
& g^{N}=\left[\begin{array}{ll}
g_{1}^{N} & g_{2}^{N} n^{N}
\end{array}\right]^{T} \text { where } g_{i}^{N} \text { is } \\
& \text { defined as } g_{i}^{N}=\max _{x} \quad g_{i}(x)
\end{aligned}
$$

A point in the design space where all the objectives are simultaneously at their worst values

\section{Problem PUi}

$$
\min _{x}\left\{g_{i}(x)\right\} \quad(1 \leq i \leq n)
$$

subject to:

$$
\begin{gathered}
f_{j}(x) \leq 0 \quad(1 \leq j \leq r) \\
h_{k}(x)=0 \quad(1 \leq k \leq s) \\
x_{l} \leq x_{i} \leq x_{u} \quad\left(1 \leq i \leq n_{x}\right)
\end{gathered}
$$

\section{Normal Constraint Method}

The NC method is based on the design space reductions using reduction constraints. The reduction constraint is constructed by ensuring the orthogonality by constructing the dot product between the normal $\vec{w}$ and $r_{0}$ an arbitrary point on a plane. The vector equation of a plane is expressed as

$$
\vec{w} \cdot\left(r-r_{0}\right)=0
$$

To solve for multi-objective solutions, a reduced feasible space is constructed using the above equation as

$$
\vec{w} \cdot(r-g) \leq 0
$$

where $g$ is any point in the feasible space. Figure $2 \mathrm{a}$ shows the non-normalized design space and the Pareto frontier of a bi-objective problem. Figure $2 b$ represents the normalized Pareto frontier in the normalized design space. In the normalized objective space, all anchor points are one unit away from the 

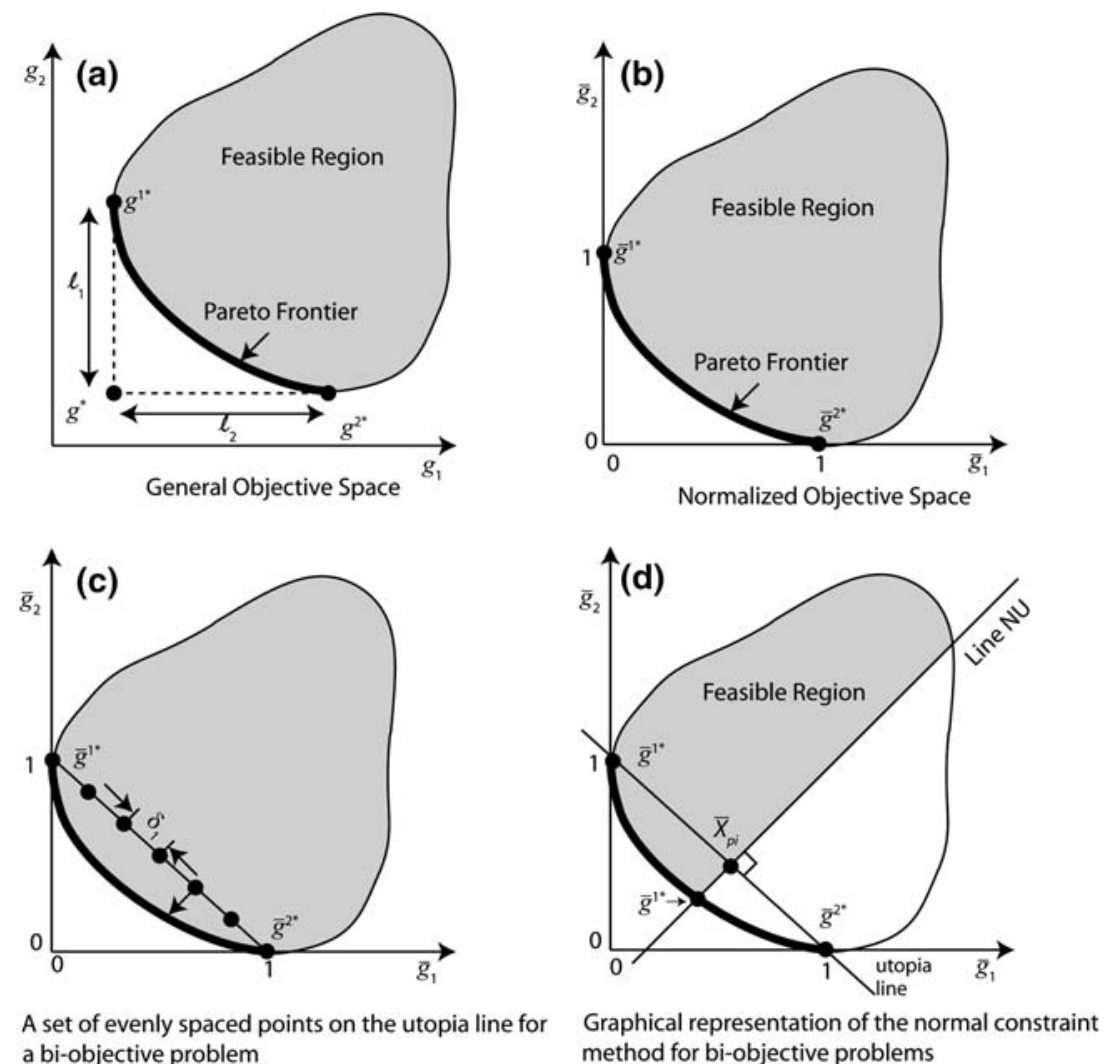

A set of evenly spaced points on the utopia line for a bi-objective problem method for bi-objective problems

FIGURE 2. Steps involved for obtaining bi-objective Pareto-optimal solutions using the Normalized Normal Constraint method for minimization. The mutually orthogonal axes $g_{1}$ and $g_{2}$ represent the individual design objectives. (a) Pareto frontier for a minimization problem and the anchor points obtained using non-linear optimization; (b) the usage of anchor points to work in a normalized objective space; (c) drawing the utopia line and constructing evenly spaced points on the utopia line; (d) constructing the normal on the utopia line and reducing the feasible space.

utopia point, and the utopia point is at the origin. A bar over a variable implies that it is normalized. The two anchor points denoted by $g_{1}^{*}$ and $g_{2}^{*}$, are obtained by successively minimizing the first and second design metrics (Problem PUi) by solving Problem PU1 and PU2 respectively. The line joining these two points is the utopia line. The actual optimization takes place in the normalized design metric space. Let $\bar{g}$ be the normalized form of $g$ and $g^{u}$, the utopia point defined as

$$
g^{u}=\left[g_{1}\left(x^{1 *}\right) g_{2}\left(x^{2 *}\right)\right]^{T}
$$

and $\ell_{1}$ and $\ell_{2}$ be the distances between $g^{2 *}$ and $g^{1 *}$, and the Utopia point, $g^{u}$, respectively (Fig. 2a). Then

$$
\begin{aligned}
& \ell_{1}=g_{1}\left(x^{2 *}\right)-g_{1}\left(x^{1 *}\right) \\
& \ell_{2}=g_{2}\left(x^{1 *}\right)-g_{2}\left(x^{2 *}\right)
\end{aligned}
$$

The normalized design objectives can then be evaluated as

$$
\bar{g}^{T}=\left[\frac{g_{1}(x)-g_{1}\left(x^{1 *}\right)}{\ell_{1}} \frac{g_{2}(x)-g_{2}\left(x^{2 *}\right)}{\ell_{2}}\right]
$$

$\bar{N}_{1}$ is defined as the direction from $\bar{g}^{1 *}$ to $\bar{g}^{2 *}$, yielding

$$
\bar{N}_{1}=\bar{g}^{2 *}-\bar{g}^{1 *}
$$

Next, the utopia line is divided into $m_{1}-1$ segments, resulting in $m_{1}$ points. A normalized increment, $\delta_{1}$ along the direction $\bar{N}_{1}$ for a prescribed number of solutions, $m_{1}$, is obtained as

$$
\delta_{1}=\frac{1}{m_{1}-1}
$$

As seen in Fig. 2b, the next step involves generating a set of evenly distributed points on the utopia line as

$$
\bar{X}_{P j}=\alpha_{1 j} \bar{g}^{1 *}+\alpha_{2 j} \bar{g}^{2 *}
$$

where

$$
0 \leq \alpha_{1 j} \leq 1
$$

$$
\sum_{k=1}^{2} \alpha_{k j}=1
$$


and $\alpha_{1 j}$ is incremented by $\delta_{1}$ between 0 and 1 (Fig. 2c), with values of $j$ as $j \in\left\{1,2, \ldots, m_{1}\right\}$.

Figure $2 \mathrm{c}$ shows one of the generic points intersecting the segments used to define a normal to the utopia line. This normal line is used to reduce the feasible space as indicated in Fig. 2d. As can be seen, if we minimize $\bar{g}_{2}$ the resulting optimum point is $\bar{g}^{2 *}$. By translating the normal line, we can see that a corresponding set of solutions will be generated. This is essentially done by generating a corresponding set of Pareto points by solving a succession of optimization runs of Problem P2. Each optimization run corresponds to a point on the utopia line. Specifically, for each generated point on the utopia line, solve for the $j$ th point.

Problem P2 (for $j$ th point)

$$
\min _{x}\left\{\bar{g}_{2}(x)\right\}
$$

subject to:

$$
\begin{gathered}
f_{j}(x) \leq 0 \quad(1 \leq j \leq r) \\
h_{k}(x)=0 \quad(1 \leq k \leq s) \\
x_{l} \leq x_{i} \leq x_{u} \quad\left(1 \leq i \leq n_{x}\right)
\end{gathered}
$$

$$
\bar{N}_{1}\left(\bar{g}-\bar{X}_{P j}\right)^{T} \leq 0
$$

This results in a set of vectors for the design parameters, one vector $x$ for each Pareto point. Then, design objectives are computed by evaluating the non-normalized design metrics that correspond to each Pareto point. The nonnormalized design objectives can be obtained through an inverse mapping of Eq. (16) by using the relation

$$
g=\left[\bar{g}_{1} \ell_{1}+g_{1}\left(x^{1 *}\right) \bar{g}_{2} \ell_{2}+g_{2}\left(x^{2 *}\right)\right]^{T}
$$

Importantly, we note that the generation of the set of Pareto points is performed in the normalized objective space, which results in critically beneficial scaling properties. Since some of the points generated in some pathological cases will be dominated by other points in the set, we use a Pareto filter (Table S1) to finally compute the true Pareto-optimal solutions. This filter compares a point generated on the Pareto frontier with every other generated point. If a point is not globally Pareto, it is discarded. The steps involved and the essential mathematical formulation for the NC method for an $n$-objective case are presented in Table 4.

TABLE 4. Normalized Normal Constraint Method for $n$-objective.

STEP Functions Formulations

1 Anchor points

Obtain the anchor points, $g^{i \star}$ for $i \in\{1,2, \ldots n\}$, by solving Problem PUi. Define hyperplane, as the one that comprises all the anchor points. This plane is called the utopia hyperplane (or, utopia plane)

2 Objectives mapping/ normalization

Compute the Nadir points and Utopia Points. Define $L$ as $L=\left[\ell_{1} \ell_{2} \ldots \ell_{n}\right]^{T}=g^{N}-g^{u}, t$ hat leads to the normalized design metrics as $\bar{g}_{i}=\frac{g_{i}-g_{i}\left(x^{*}\right)}{\ell_{i}} \quad i=1,2, \ldots, n$,

3

Utopia plane vector

Define the direction, $\bar{N}_{k}$ from $\bar{g}^{k *}$ to $\bar{g}^{n *}$ for $k \in\{1,2, \ldots, n\}$ as $\bar{N}_{k}=\bar{g}^{n *}-\bar{g}^{k *}$

$4 \quad$ Normalized increments Compute a normalized increment, $\delta_{k}$ along the direction $\bar{N}_{k}$ for a prescribed number of solutions, $m_{k}$, along the associated $\bar{N}_{k}$ direction: $\delta_{k}=\frac{1}{m_{k}-1} \quad(1 \leq k \leq n-1)$

$5 \quad$ Generate utopia hyperplane points

6

Pareto points generation
Evaluate a set of evenly ${ }_{n}$ distributed points on the Utopia hyperplane as $\bar{X}_{p j}=\sum^{n} \alpha_{k j} \bar{g}^{k *}$ where $0 \leq \alpha_{k j} \leq 1$ and $\sum \alpha_{k j}=1$. Figure $1 c$ describes how generic points are ${ }^{k}$ generated in the utopia plane, where two planes serve as constraints. Figure $2 c$ shows the resulting uniformly distributed points on the utopia plane for a two-dimensional case in the normalized objective space

A set of well-distributed Pareto solutions in the normalized objective space. For each value of $\bar{X}_{p j}$ generated in Step 5, the corresponding Pareto solution is obtained by solving the following problem:

Problem $\mathrm{Pn}$ $\min _{x}\left\{\bar{g}_{n}(x)\right\}$

Subject to:

$$
\begin{array}{cc}
f_{j}(\boldsymbol{x}) \leq 0 & (1 \leq j \leq r) \\
h_{k}(\boldsymbol{x})=0 & (1 \leq k \leq s) \\
x_{l} \leq \boldsymbol{x}_{i} \leq \boldsymbol{x}_{u} & \left(1 \leq i \leq n_{x}\right) \\
\bar{N}_{k}\left(\bar{g}-\bar{X}_{P j}\right)^{T} \leq 0 & (1 \leq k-1)
\end{array}
$$

$7 \quad$ Pareto design metrics values
The design metrics values for the Pareto solutions obtained in Step 6 can be obtained using the equation $g_{i}=\bar{g}_{i} \ell_{i}+g_{i}\left(x^{i *}\right), \quad i=1,2, \ldots, n$ 


\section{Normal Constraint Energy and Flux Balance Analysis (NCEFBA)}

This section combines FBA, EBA, and NC constraints. In the combined EBA and FBA, non-linear thermodynamic constraints analogous to electrical circuit system constraints are included with the linear FBA constraints. The addition of non-linear thermodynamic constraints leads to a non-linear optimization problem. To avoid repetition in the presented NC method we will show here only the fluxes that are changed in the previously presented NC. There are changes in anchor points which lead to a different utopian hyperplane. Further, both FBA and EBA constraints are added to the NC formulation with the optimized quantity being the desired fluxes as objectives.

Computation of the Utopia hyperplane: The anchor points for NCEFBA are obtained by solving the Problem PUi, which is now defined as follows:

Problem PUi

$$
\min _{x}\left\{g_{i}(x)\right\} \quad(1 \leq i \leq n)
$$

subject to:

$$
\begin{gathered}
S_{u} J_{u}=-S_{m} J_{m} \\
K^{T} \Delta \mu=K^{T} S^{T} \mu=0 \\
J=J_{+}-J_{-} \\
-J^{j} \Delta \mu^{j}=-R T\left(J_{+}^{j}-J_{-}^{j}\right) \ln \left(\frac{j_{-}^{j}}{J_{+}^{j}}\right) \geq 0 \\
h d r=-J^{T} \Delta \mu>0 \\
(h d r)_{l b} \leq h d r \leq(h d r)_{u b} \\
J_{l b} \leq J \leq J_{u b} \\
0 \leq J_{+} \leq \infty \\
0 \leq J_{-} \leq \infty \\
J_{l b}^{e x t} \leq J^{e x t} \leq J_{u b}^{e x t} \\
\Delta \mu_{l b} \leq \Delta \mu \leq \Delta \mu_{u b}
\end{gathered}
$$

where vector $x$ is defined as

$$
x^{T}=\left[\begin{array}{llll}
J^{T} & \Delta \mu^{T} & J_{+}^{T} & J_{-}^{T}
\end{array}\right]
$$

and the boundary constraints are meant to be satisfied component-wise.
Computation of Pareto points: Once anchor points are obtained, a set of well-distributed Pareto solutions are generated in the normalized objective space, by solving Problem Pn:

Problem Pn (for $j$ th point)

$$
\min _{x}\left\{\bar{g}_{n}(x)\right\}
$$

subject to:

$$
\begin{gathered}
S_{u} J_{u}=-S_{m} J_{m} \\
K^{T} \Delta \mu=K^{T} S^{T} \mu=0 \\
J=J_{+}-J_{-} \\
-J^{j} \Delta \mu^{j}=-R T\left(J_{+}^{j}-J_{-}^{j}\right) \ln \left(\frac{J_{-}^{j}}{J_{+}^{j}}\right) \geq 0 \\
h d r=-J^{T} \Delta \mu>0 \\
(h d r)_{l b} \leq h d r \leq(h d r)_{u b} \\
J_{l b} \leq J \leq J_{u b} \\
0 \leq J_{+} \leq \infty \\
0 \leq J_{-} \leq \infty \\
J_{l b}^{e x t} \leq J^{e x t} \leq J_{u b}^{e x t} \\
\Delta \mu_{l b} \leq \Delta \mu \leq \Delta \mu_{u b} \\
\left.\bar{X}_{P j}\right)^{T} \leq 0 \quad(1 \leq n-1)
\end{gathered}
$$

where vector $x$ is defined as

$$
x^{T}=\left[\begin{array}{llll}
J^{T} & \Delta \mu^{T} & J_{+}^{T} & J_{-}^{T}
\end{array}\right]
$$

and the boundary constraints are meant to be satisfied component-wise.

The combined algorithm NCEFBA was implemented in MATLAB (Mathworks Inc.) and all simulations were run on a PENTIUM 3.0 GHZ dual processor. For whole genome-scale applications the presented algorithm can be easily implemented using parallel computing in High Performance Fortran 90. ${ }^{16}$

\section{Hepatic Function Specific Fluxes for Pareto Optimization}

The main goal that we wish to achieve in the BAL device is for hepatocytes to perform at the highest level of liver-specific functions. Therefore, for hepatic 
metabolic optimization, the set of objective functions maximizing urea, albumin, NADPH, and glutathione synthesis, ATP generation are chosen. NADPH, which is produced in the pentose phosphate pathway (PPP), is primarily used in non-proliferating hepatocytes for cytochrome p450 dependent oxidation reactions (detoxification reactions) and glutathione synthesis. Hence, to increase the NADPH flux, the NADPHgenerating oxidative branch of the PPP represented in a lumped fashion as reaction 46 (Table 1) is maximized. As a marker of secretory liver-specific function, we use albumin synthesis, which is maximized by modulating flux 47 . Urea synthesis is primarily derived from ammonia and aspartate generated through transamination reactions and is maximized by modulating reaction 16. The tripeptide glutathione $(\mathrm{GSH}$, $\gamma$-Glu-Cys-Gly) is an important reductant and has many detoxifying and cytoprotective effects. The synthesis of glutathione is increased by maximizing reaction 48. The ATP generation is maximized by increasing the TCA cycle fluxes $(11,43,44)$. Figure 3 presents the comprehensive hepatic metabolic network with all the cycles shown with each reaction and the constraints for this metabolic network are listed in Table S2. The complexity of the hepatic metabolic network shown in Fig. 3, is due to the various

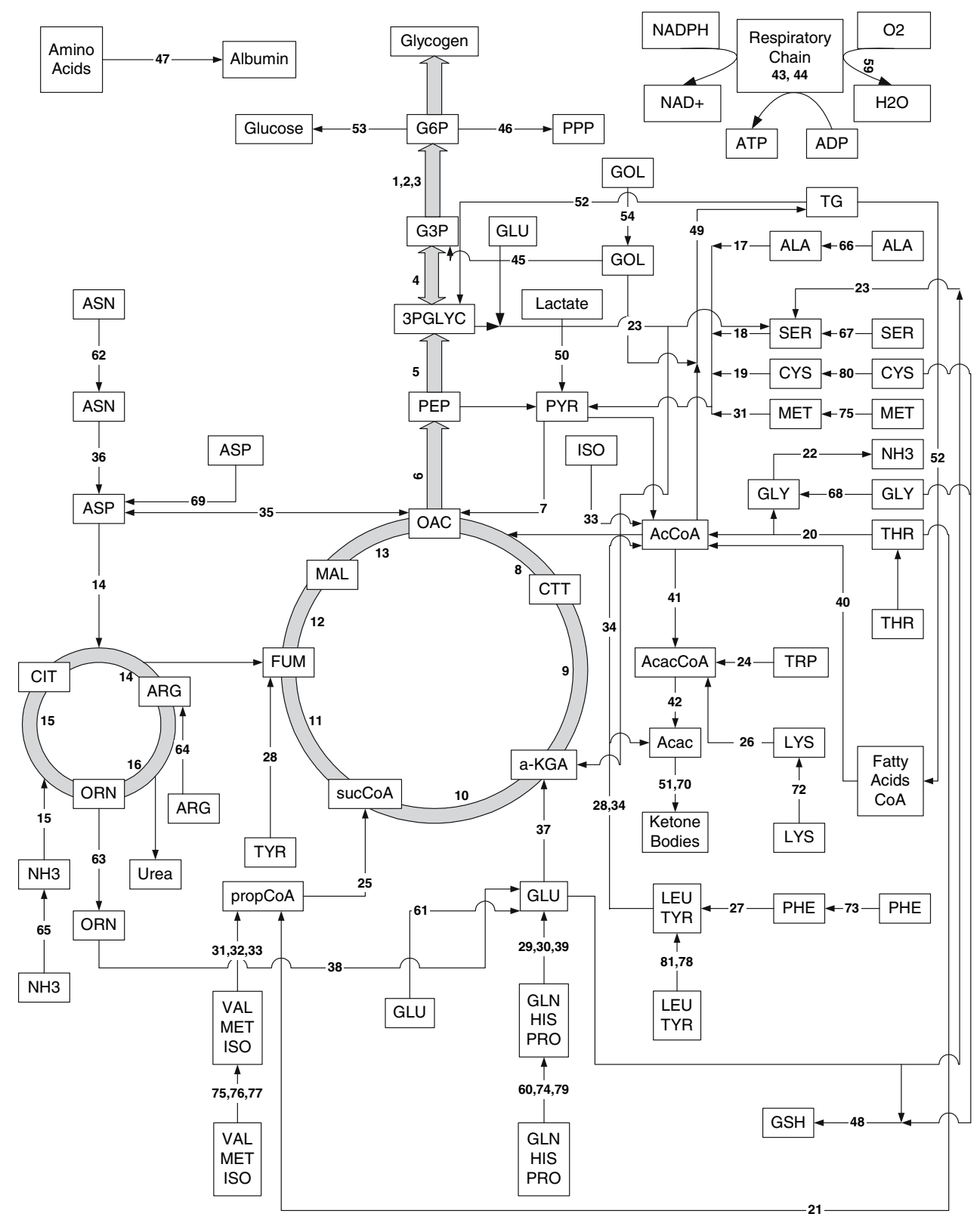

FIGURE 3. Hepatic metabolic network showing the linkages among the various metabolites. 
inter-connected cycles (urea cycle linked with TCA cycle; TCA cycle linked with fatty acids oxidation; linkage of Pentose Phosphate Pathway with both gluconeogenesis and glycolysis) and the dependence of hepatocyte specific objectives (albumin synthesis, urea secretion, NADPH synthesis, ATP generation, glutathione synthesis) on all of the cycles. Specifically, any change induced in one objective alters the availability of substrates for other objectives resulting in a tradeoff or competing behavior in various objectives. It is important to emphasize that the more branched the network, the higher the tradeoff between various objectives.

\section{RESULTS}

Pareto-optimal solutions are accepted solutions of multi-objective optimization problems, and can serve as a useful tool to understand the underlying tradeoffs between conflicting design objectives and cellular phenotypes. Pareto optimality analysis has been applied to numerous disciplines and more recently to
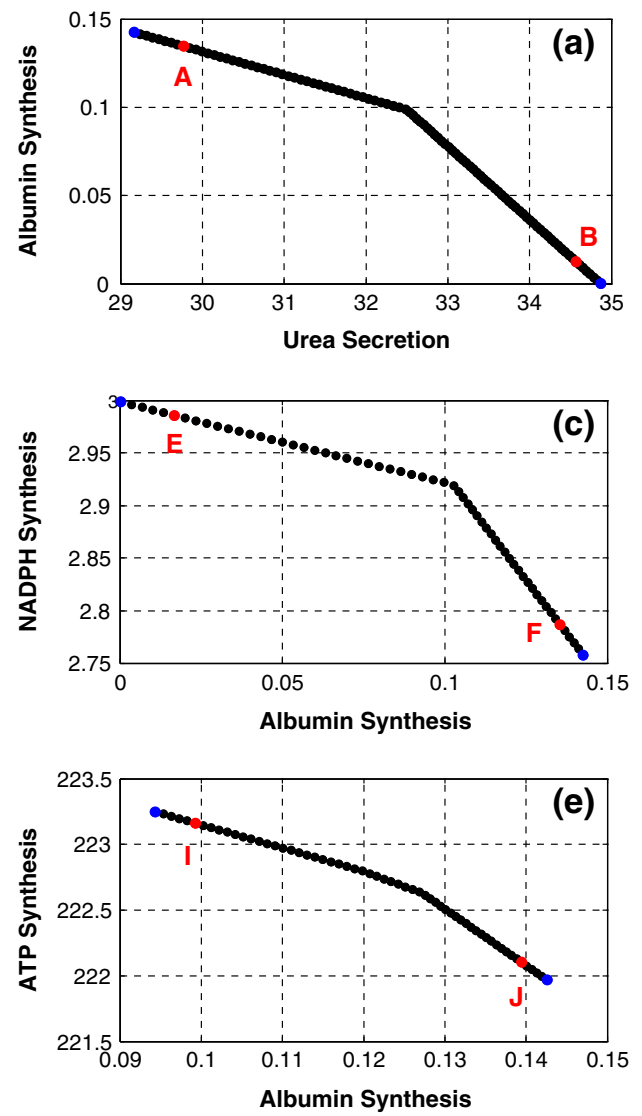

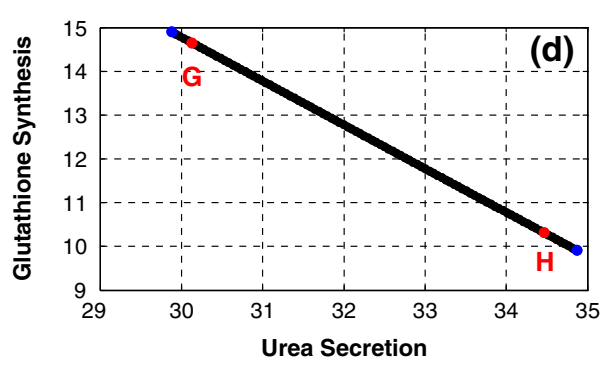

cellular systems. ${ }^{22}$ As mentioned earlier, usage of FBA alone can lead to thermodynamically infeasible fluxes. Consequently, we chose to combine both FBA and EBA constraints with Pareto optimality to optimize hepatocellular function in the context of a BAL device. As part of this analysis, we first obtained Pareto frontiers between various bi-objective combinations of liver-specific functions (albumin synthesis, urea secretion, NADPH synthesis, GSH synthesis, and ATP generation). This was done for hepatocytes in a gluconeogenic state and in a glycolytic state. Next, for a representative case, i.e., ATP generation vs. urea secretion, we compared the Pareto frontier using NCEBFBA (i.e., both FBA and EBA) with FBA alone. Lastly, we obtained the Pareto solutions in the presence of measurement constraints. The experimentally measured flux data for gluconeogenic and glycolytic state were taken from Chan et al., ${ }^{5,6}$ respectively.

\section{Pareto Frontiers of Liver-Specific Functions}

Pareto optimality analysis here is carried out first in gluconeogenic hepatocytes (Fig. 4) and then for
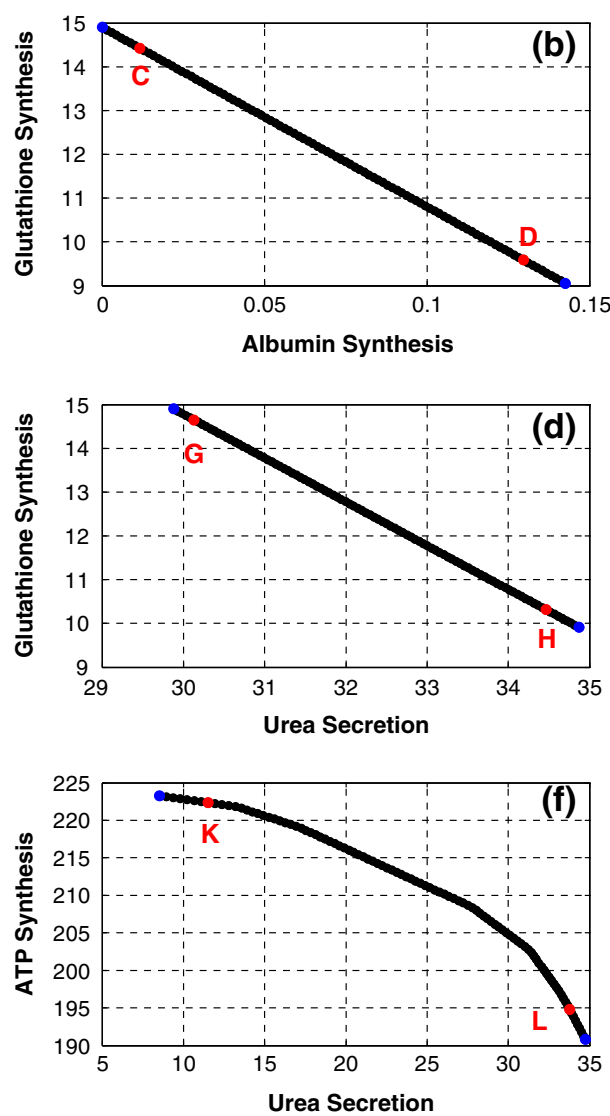

FIGURE 4. Pareto frontiers for bi-objective problems in hepatocytes operating in a gluconeogenic mode. Five major hepatic functions were considered: albumin, urea, ATP, NADPH, and glutathione synthesis. (a) Albumin vs. urea synthesis; (b) glutathione vs. albumin synthesis; (c) NADPH vs. albumin synthesis; (d) glutathione vs. urea synthesis; (e) ATP vs. albumin synthesis; (f) ATP vs. urea synthesis. The blue circles are the anchor points, black circles are Pareto-optimal solutions, and red circles labeled A to $L$ are selected Pareto solutions for which flux distributions are shown in Table S3. 
glycolytic hepatocytes (Fig. 6). This distinction was necessary because the hepatic metabolic network used in each case is different. Note that in both figures, the same panels analyze the same objectives to facilitate the comparison of results obtained in the gluconeogenesis and glycolysis modes. For each Pareto curve shown in Fig. 4 for gluconeogenic hepatocytes, Fig. 5 shows the relative flux changes that are necessary when switching objective priority. This information is summarized in Table 5, where important groups are clustered together. Similarly, for each Pareto curve shown in Fig. 6 for glycolytic hepatocytes, Fig. 7 and Table 6 summarize the flux changes that are necessary when switching objective priority. Supplementary Tables S3 and $\mathrm{S} 4$ provide the comprehensive set of flux data that are summarized in Figs. 4 and 6, respectively.
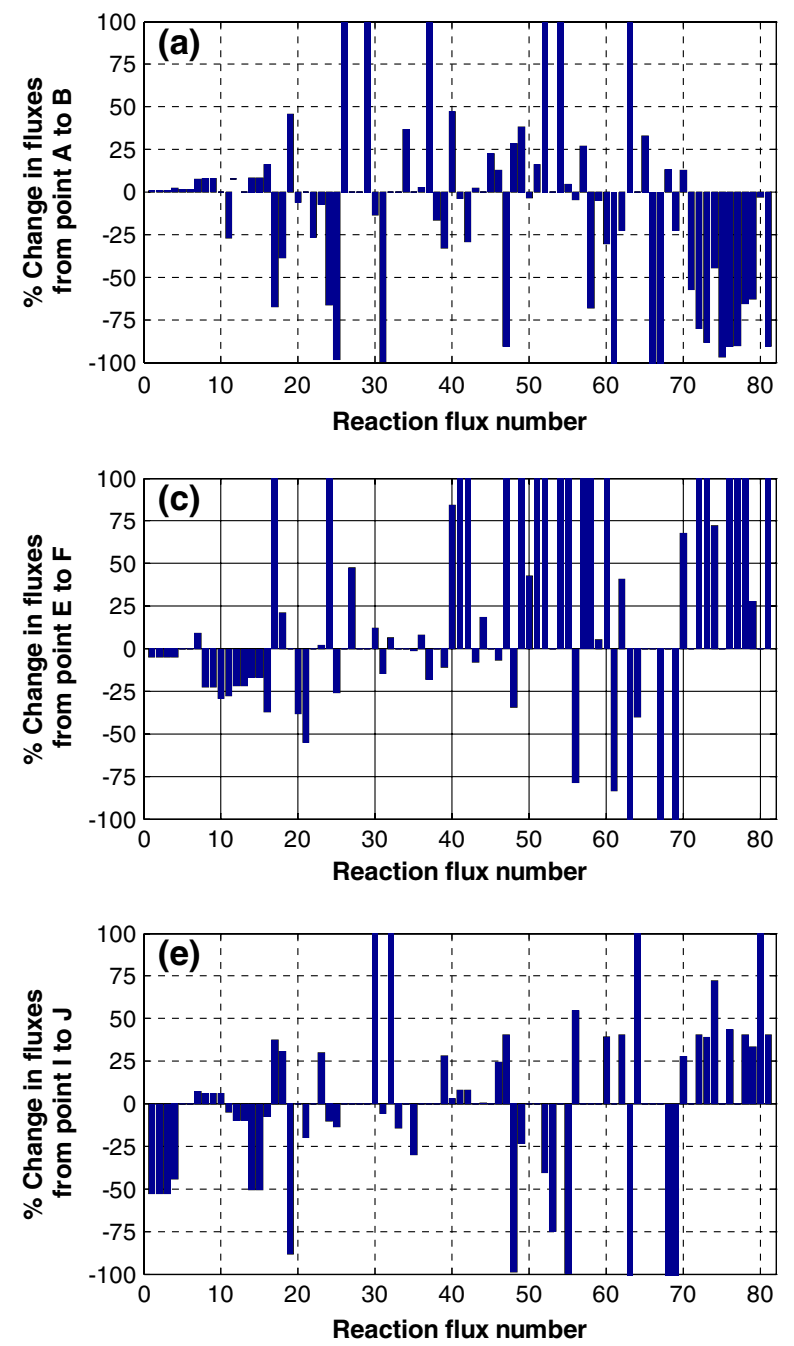

The bi-objective Pareto-optimal solutions were first obtained using the NCEFBA approach for various binary combinations of liver-specific objectives in gluconeogenic hepatocytes (Fig. 4). The Pareto frontiers for albumin synthesis vs. urea secretion, glutathione synthesis vs. albumin synthesis, NADPH synthesis vs. albumin synthesis, glutathione synthesis vs. urea secretion, ATP generation vs. albumin synthesis, and ATP generation vs. urea secretion are shown in Figs. 4a-4f, respectively.

As seen in Fig. 4, all of these objectives have a tradeoff region with each other. For example, we cannot have both albumin and urea synthesis at their maximal values. Additionally, there is a tradeoff between other liver-specific functions such as GSH and albumin synthesis, NADPH and albumin
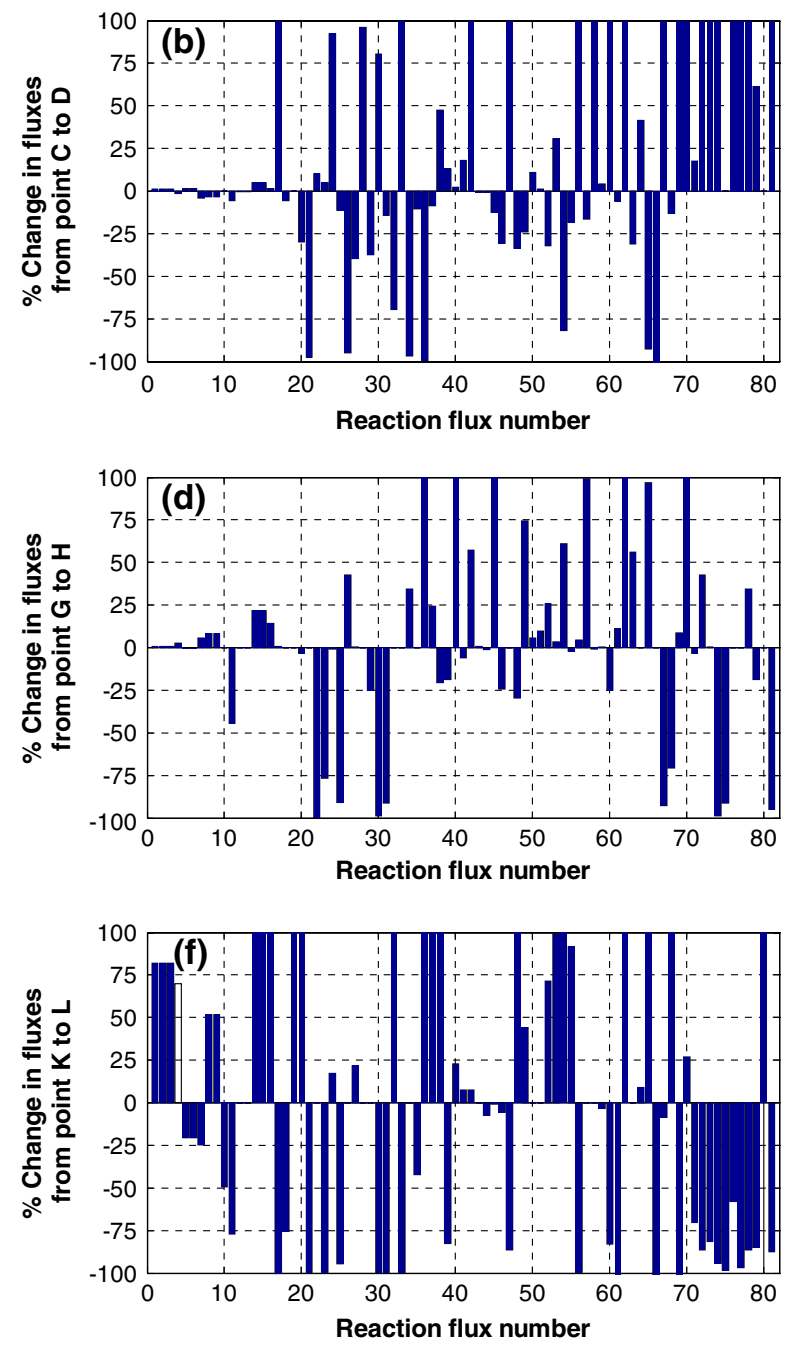

FIGURE 5. Distribution of flux changes when moving along the Pareto surface in Fig. 4: (a) \% Flux changes from point $A$ to point $B$ in Fig. 4a; (b) \% Flux changes from point C to point D in Fig. 4b; (c) \% Flux changes from point $E$ to point $F$ in Fig. 4c; (d) \% Flux changes from point $\mathrm{G}$ to point $\mathrm{H}$ in Fig. $4 \mathrm{~d}$; (e) \% Flux changes from point I to point $\mathrm{J}$ in Fig. $4 \mathrm{e}$; (f) \% Flux changes from point $\mathrm{K}$ to point $L$ in Fig. 4f. The corresponding flux values are in Table S3. Note that the \% flux changes for all figures are on $y$-axis and the corresponding reaction flux number is shown on the horizontal axis. Only changes up to $100 \%$ are shown in the figure. 


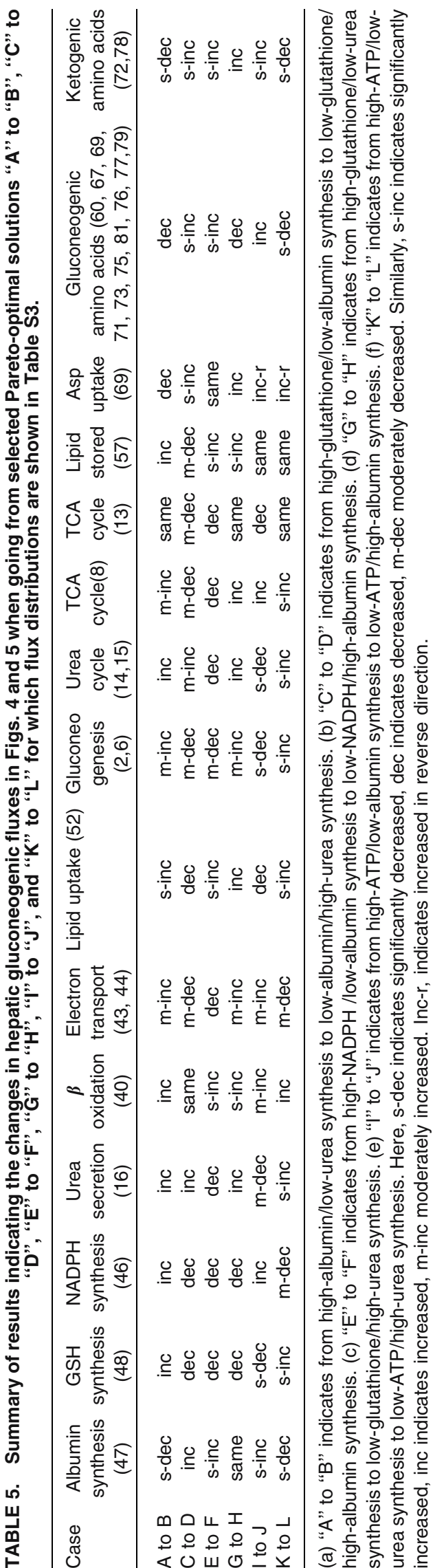

synthesis, GSH synthesis and urea secretion, ATP synthesis and albumin synthesis, and ATP synthesis and urea secretion. As seen in these figures, the tradeoff region or range of Pareto-optimal solutions (how far the optimal value is from the "anchor value") for albumin synthesis is very high compared to NADPH, GSH and ATP synthesis and urea secretion. Several other combinations were also tested and all of them indicated Pareto optimality between various objectives (data not shown). Figure 5 presents the metabolic flux profiling of Pareto-optimal fluxes throughout the tradeoff region, which shows the changes required in flux values and direction (i.e., increasing or decreasing) as the objective preference is changed from one objective to another. The corresponding flux values for these cases are presented in Table S3.

The Pareto frontiers for various binary combinations of objectives were also obtained for glycolytic hepatocytes (Fig. 6). As in the case of gluconeogenic hepatocytes, these objectives have a tradeoff region with each other, and some objectives change over a wide range (e.g., albumin, urea, and GSH) while some change only little (NADPH and ATP). Figure 7 presents the metabolic flux profiling of Pareto-optimal fluxes throughout the tradeoff region, which shows the changes required in flux values and direction (i.e., increasing or decreasing) as the objective preference is changed from one objective to another. The corresponding flux values for these cases are presented in Table S4.

Figure $4 \mathrm{a}$ examines the tradeoff between albumin and urea secretion in gluconeogenic hepatocytes. Many flux changes were required to go from Paretooptimal solutions "A" to "B" in Fig. 4a, in other words, when going from a state of high-albumin/lowurea secretion rate to a low-albumin/high-urea secretion rate. As seen in Fig. 5a and summarized in Table 5, this change required increasing marginally gluconeogenic fluxes (1-9), increasing moderately urea cycle fluxes (14-15), decreasing formation of glutamate (38-39), increasing oxidation of triglycerides (52), decreasing uptake of both glucogenic (proline, 60; serine, 67; aspartate, 69; threonine, 71; phenylalanine, 73; methionine, 75; valine, 76; isoleucine, 77; glutamine, 79, tyrosine, 81) and ketogenic (lysine, 72; leucine, 78) amino acids, with the exception of glycine (34) uptake, which was increased. Arginine (64) uptake rate did not change because it was at its maximum at both optimal points. Histidine (18) uptake increased because it results in an increase of $\alpha$-ketoglutarate. The uptake of pyruvate-forming amino acids (alanine, 66; serine, 67; and threonine, 71), fumarate-forming amino acids (phenylalanine, 73; and tyrosine, 81), and succinyl CoA-forming amino acids (methionine, 75; valine, 

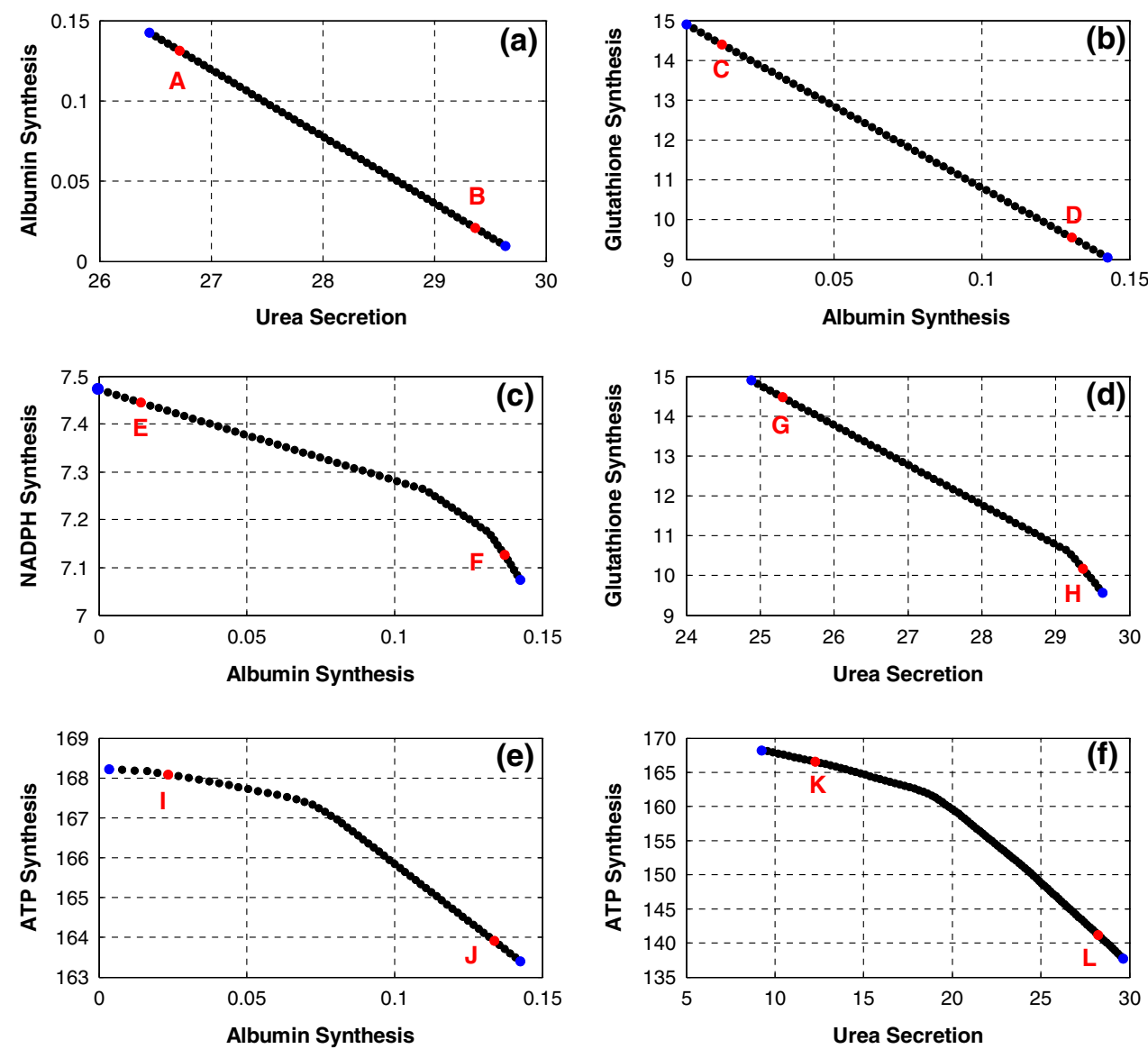

FIGURE 6. Pareto frontiers for bi-objective problems in hepatocytes operating in a glycolysis mode. Five major hepatic functions were considered: albumin, urea, ATP, NADPH, and glutathione synthesis. (a) Albumin vs. urea synthesis; (b) glutathione vs. albumin synthesis; (c) NADPH vs. albumin synthesis; (d) glutathione vs. urea synthesis; (e) ATP vs. albumin synthesis; (f) ATP vs. urea synthesis. The blue circles are the anchor points, black circles are Pareto-optimal solutions, and red circles labeled $A$ to $L$ are selected Pareto solutions for which flux distributions are shown in Table S4.

76; and threonine, 71), which play a major role in albumin synthesis, were decreased.

When considering the tradeoff between albumin and urea secretion in glycolytic hepatocytes (Figs. 6a and $7 \mathrm{a})$, the main difference with the case of gluconeogenic hepatocytes was in the $\beta$-oxidation flux which was higher in gluconeogenesis and decreased in glycolysis. This is expected because glycolysis is dominant in the fed state and gluconeogenesis in the fasted state. Further, the production of ketone bodies through $\beta$-oxidation occurs mostly in the fasted state.

Next, we investigated the tradeoff between glutathione and albumin synthesis in gluconeogenic hepatocytes. The Pareto curve is shown in Fig. 4b. Going from Pareto-optimal solutions " $C$ " to " $\mathrm{D}$ " also required many flux changes, which are reported in Fig. 5b and Table 5. There was a marginal increase in urea cycle fluxes (14-15), a decrease in lipid uptake (52) and lipid stored (57), and a significant increase in aspartate uptake (69). Additionally, the uptake of both gluconeogenic amino acids $(60,67,69,71,73,76,77$,
79, and 81) and ketogenic amino acids $(72,78)$ increased. The corresponding flux values for NADPH synthesis decreased. There were no significant differences in the results of this analysis when considering glycolytic hepatocytes (Figs. 6b and 7b, and Table 6).

Considering the tradeoff between NADPH synthesis and albumin synthesis (Figs. 4c and 6c), flux changes required to move from points $\mathrm{E}$ to $\mathrm{F}$ along the Pareto frontier were generally similar in both gluconeogenic and glycolytic hepatocytes (Figs. 5c and 7c), with the exception of $\beta$-oxidation, electron transport (43, 44), lipid uptake and lipid storage fluxes. This is because fatty acid synthesis significantly consumes NADPH (14 molecules of NADPH per molecule of palmitate).

Considering the tradeoff between glutathione synthesis and urea secretion (Figs. $4 \mathrm{~d}$ and $6 \mathrm{~d}$ ), the changes in flux required to move from points $\mathrm{G}$ to $\mathrm{H}$ along the Pareto frontier were also generally similar in both gluconeogenic and glycolytic hepatocytes (Figs. 5d and $7 d$ ), with the exception of aspartate uptake (69). 

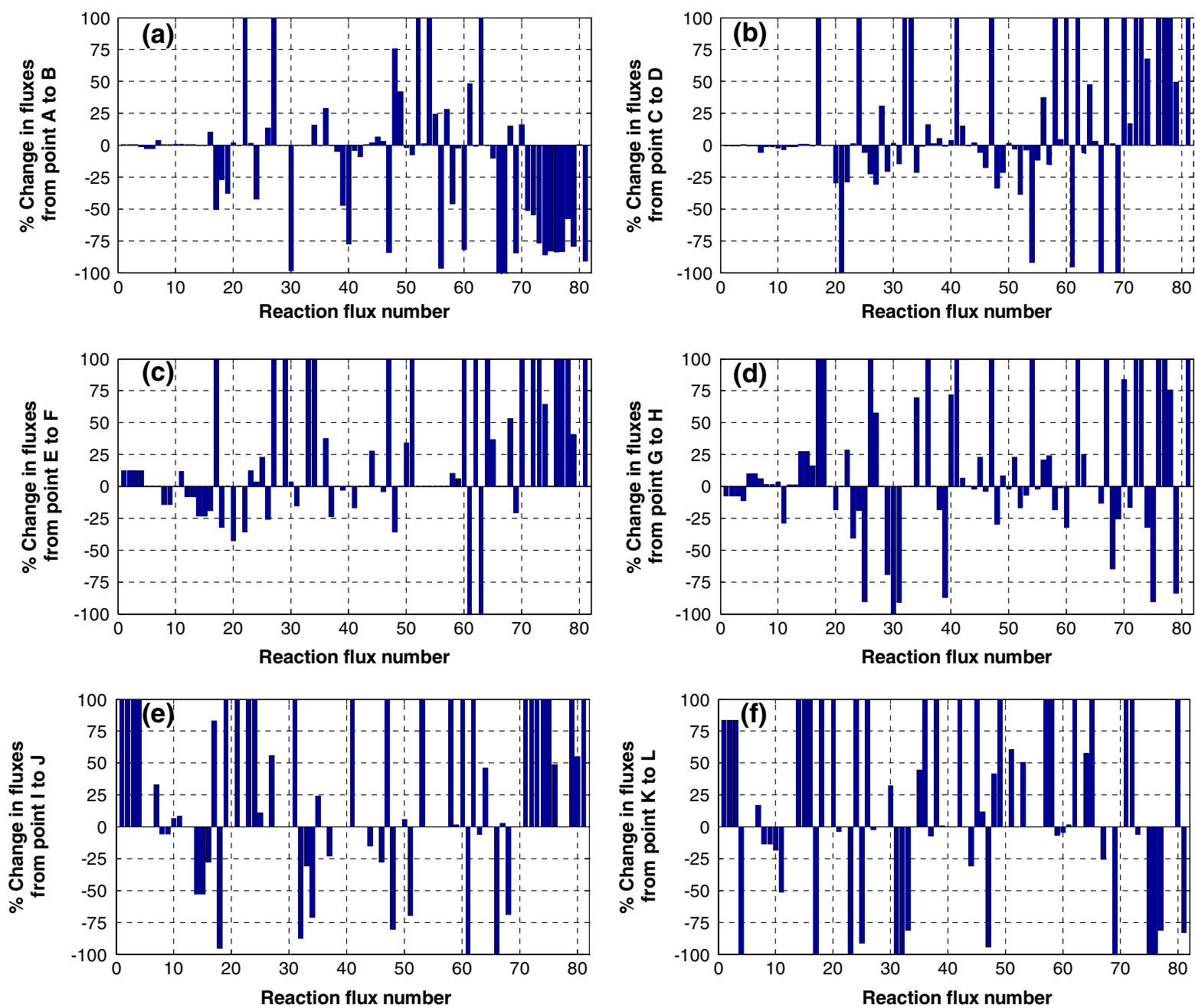

FIGURE 7. Distribution of flux changes when moving along the Pareto surface in Fig. 6: (a) \% Flux changes from point $A$ to point $B$ in Fig. 6a; (b) \% Flux changes from point $C$ to point $D$ in Fig. $6 b$; (c) \% Flux changes from point $E$ to point $F$ in Fig. $6 c$; (d) \% Flux changes from point $\mathrm{G}$ to point $\mathrm{H}$ in Fig. $6 \mathrm{~d}$; (e) \% Flux changes from point I to point $\mathrm{J}$ in Fig. 6e; (f) \% Flux changes from point $\mathrm{K}$ to point $L$ in Fig. 6f. The corresponding flux values are in Table S4. Note that the \% flux changes for all figures are on $y$-axis and the corresponding reaction flux number is shown on the horizontal axis. Only changes up to $100 \%$ are shown in the figure.

Considering the tradeoff between ATP synthesis and albumin synthesis (Figs. 4e and 6e), the changes in flux required to move from points $\mathrm{I}$ to $\mathrm{J}$ along the Pareto frontier significantly differed between gluconeogenic and glycolytic hepatocytes (Figs. 5e and 7e), mainly with respect to gluconeogenesis fluxes (2-6), and TCA cycle fluxes (8-13). In the gluconeogenesis mode, TCA cycle fluxes are higher because of increased demand to produce ATP (gluconeogenesis consumes ATP too), since glycolysis itself produces ATP (2 molecules of ATP for 1 molecule of glucose consumed).

Considering the tradeoff between ATP synthesis and urea synthesis (Figs. 4f and 6f), the changes in flux required to move from points $\mathrm{K}$ to $\mathrm{L}$ along the Pareto frontier in both gluconeogenic and glycolytic hepatocytes were mainly lipid uptake (52), TCA cycle (8), aspartate uptake (69) and the uptake of gluconeogenic and ketogenic amino acids. This is expected because higher urea secretion could be achieved with increased uptake of arginine or aspartate under gluconeogenic conditions. Higher urea secretion has been seen to require an increase in gluconeogenic fluxes and this is coupled with an increase in TCA cycle fluxes, which necessitates an increase in aspartate uptake.

\section{Effect of FBA+EBA on Pareto Frontier Compared to FBA Alone}

We compared Pareto frontiers for the representative case of ATP synthesis vs. urea secretion considering FBA (i.e., mass balance) constraints only and then both FBA and EBA (i.e., both mass balance and thermodynamic) constraints. Figure $8 \mathrm{a}$ shows the Pareto frontiers when hepatocytes are in a glycolysis 


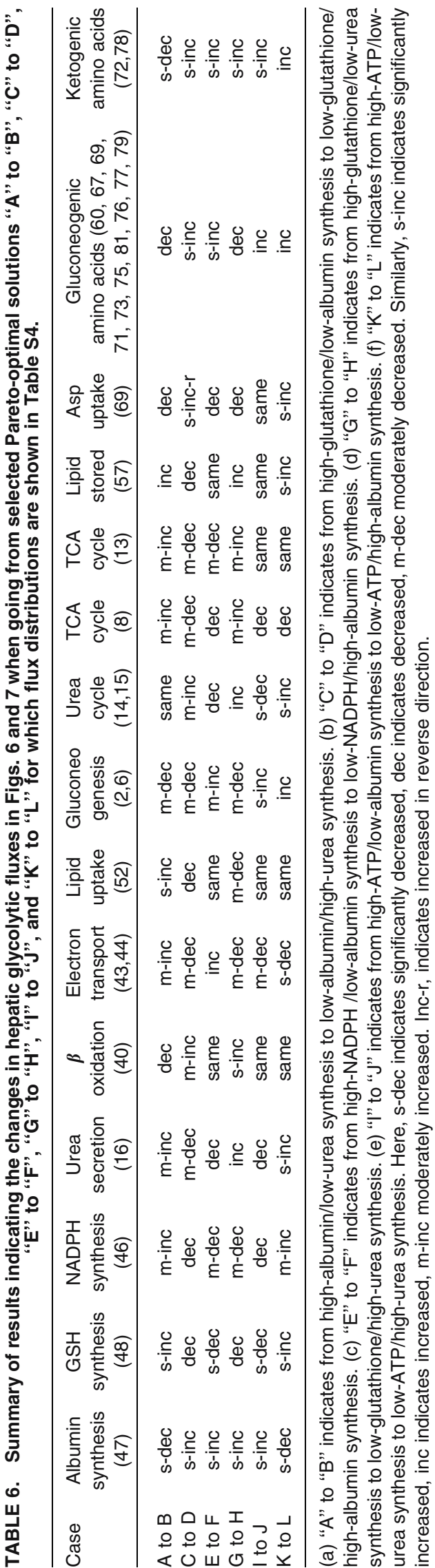

mode. The addition of EBA constraints generally reduced the feasible space of the flux distribution, and changed the Pareto frontiers accordingly. For example, for the representative case of ATP synthesis vs. urea secretion in glycolytic hepatocytes (shown in Fig. 8a), the Pareto frontier obtained using both FBA \& EBA constraints was below that obtained using FBA alone. Furthermore, the fluxes obtained using both approaches were vastly different throughout the Pareto frontier. Figures $8 \mathrm{~b}-8 \mathrm{~d}$ show the effect of adding EBA constraints on the Pareto-optimal solutions A, B, and $\mathrm{C}$, respectively, and the corresponding flux values are presented in Table S5. In all cases, EBA reduced the feasible space. It is to be noted that urea secretion (flux 16 on the abscissa) was kept constant to analyze these differences. Essentially, keeping urea secretion constant is necessary to compare the Pareto solutions obtained after different measurements.

As seen in Figs. 8b-8d several glycolytic fluxes (2-6) and catabolic fluxes that produce pyruvate $(17,18$, and 19) were changed at points A, B, and C on the Pareto frontier when adding EBA constraints. On the other hand, there was a marginal difference in TCA cycle flux (8) at Pareto solution A, and no difference at points B or C. Similarly, when going from A to C, there was a decreased difference in the uptake of succinyl CoA forming amino acids (threonine, 71, methionine, 75, and valine, 76). Notably, the difference at point $\mathrm{A}$ in the aspartate production through asparagine (36) first increases with the increased urea secretion, then the difference decreases significantly at Pareto solution B. Throughout the Pareto frontier there was a decreased ketone body production (41) when adding EBA constraints. The change in lipid uptake and lipid storage fluxes when adding EBA constraints became more prominent when going from point $\mathrm{A}$ to point $\mathrm{C}$.

\section{Effect of Measured Fluxes on Pareto Frontiers}

The incorporation of experimental measurements also reduced the feasible space for the fluxes and the Pareto-optimal solutions (Fig. 9). The control case has no measurements and is labeled as M0 (shown as black dots in Fig. 9). Experimental measurements were included as equality constraints in the stoichiometric matrix according to Eq. (3). For this analysis, four different bi-objective sets were examined as examples. Figure 9 shows the effect of adding four different measurement sets on the Pareto frontiers. Measurements were included in a sequential fashion. The Pareto curve M1 (shown as red diamonds) was obtained after adding lactate (50), glucose (53), and glutamate (61) measurements. The Pareto curve M2 (shown as blue squares) was obtained after adding to 
M1 measurements, glutamine (79) and tyrosine (81). The Pareto curve M3 (shown as yellow triangles) is obtained after adding to M2 measured fluxes, alanine (66), serine (67), and glycine (68) flux measurments. The Pareto curve M4 (shown as green stars) is obtained after adding to M3 measured fluxes, methionine flux measurement (75). Experimental data for gluconeogenesis and glycolysis were taken from (14) and (16), respectively.

We looked at four representative bi-objective sets (albumin vs. urea; ATP vs. albumin; glutathione vs. urea; and ATP vs. urea) to ascertain the changes in Pareto frontiers. The three first sets are in gluconeogenic mode and the last one is in glycolytic mode.

Figure 9a show Pareto frontiers for albumin synthesis vs. urea secretion and Fig. 9b shows the Pareto frontiers for ATP synthesis vs. albumin synthesis. In both cases, as more measured data are included, the anchor points of the Pareto frontiers move towards the center and eventually become a single point solution. Figure 9c shows that Pareto frontiers for glutathione synthesis vs. urea secretion, in the higher glutathione synthesis range did not change when including measurement sets M1 and M2, although they did when including measurement sets M3 and M4. Figure 9d shows the Pareto frontiers for ATP synthesis vs. urea secretion. Pareto frontiers were lowered when adding each measurement set. The corresponding fluxes for these four cases are presented in Table S6. Figures $10 \mathrm{a}-10 \mathrm{~h}$ show the distribution of flux changes for the cases shown in Figs. 9a-9d respectively.

When considering the albumin vs. urea case (Fig. 9a), the change in Pareto curve at high-urea secretion was associated with many differences in fluxes (Fig. 10a), including a moderate decrease in gluconeogenic fluxes (2-4), a moderate increase in TCA cycle flux (8), a decrease in urea secretion (16) and $\beta$-oxidation (40), an increase in electron transport $(43,44)$, lipid uptake (52), lipid stored (57), albumin (47), NADPH (46) and GSH (48) synthesis. The change in Pareto curve at high-albumin synthesis also caused flux changes (Fig. 10b), including a moderate increase in gluconeogenic fluxes (2-6), TCA cycle fluxes $(8,13)$, urea cycle fluxes $(14,15)$ and urea secretion (16), a significant increase in $\beta$-oxidation (40), electron transport $(43,44)$, lipid storage (57), NADPH (46) and GSH (48) synthesis, significant decrease in lipid uptake (52) and albumin synthesis (47). Additionally, there was a decrease in uptake of both gluconeogenic and ketogenic amino acids.
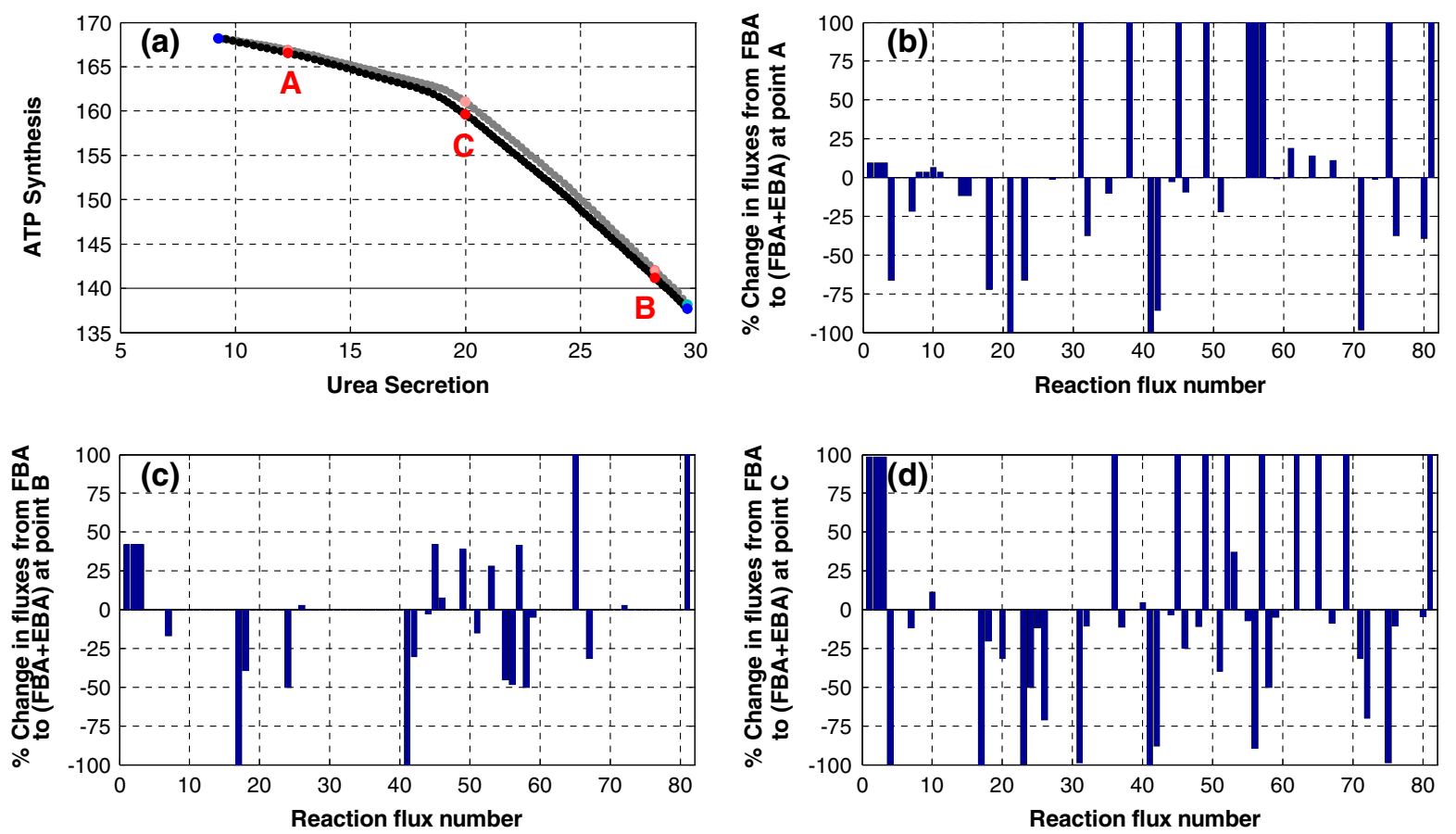

FIGURE 8. Effect of adding EBA constraints on optimal fluxes for the representative case of ATP synthesis vs. urea secretion biobjective problem in glycolytic hepatocytes. (a) Pareto frontiers using FBA constraints alone (gray line) and FBA + EBA constraints (black line). The blue circles are the anchor points and the red circles are selected Pareto solutions A, B, C for which the complete set of fluxes is provided in Table S5. (b) Distribution of flux changes when adding EBA constraints at point A. (c) Distribution of flux changes when adding EBA constraints at point $B$. (d) Distribution of flux changes when adding EBA constraints at point $\mathrm{C}$. In panels (b-d), the data are expressed as \% flux change and the corresponding reaction flux number is shown on the horizontal axis. Note that urea secretion was kept constant when analyzing these differences. 

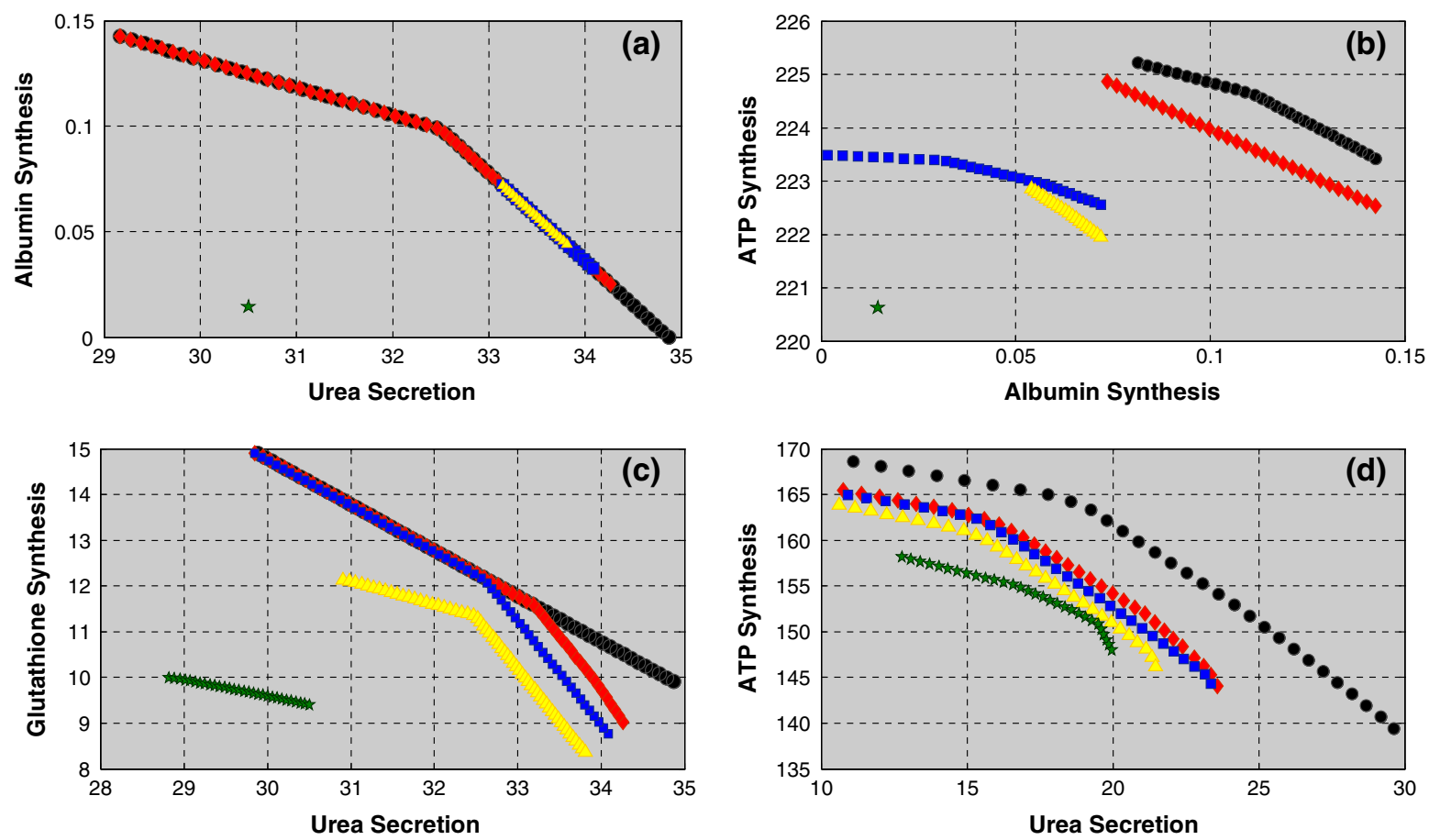

FIGURE 9. Effect of adding flux measurements to Pareto frontiers. Measurements were incorporated as equality constraints in the stoichiometric matrix. Four different bi-objective cases are shown in panels a-d, respectively: albumin vs. urea synthesis (gluconeogenesis mode), ATP vs. albumin synthesis (gluconeogenesis mode), glutathione vs. urea synthesis (gluconeogenesis mode), and ATP vs. urea synthesis (glycolysis mode). The control case has no measurements (M0 in black). The Pareto curve M1 (shown as red diamonds) is obtained after adding measured flux 50 (value of 1.0815 and 1.08 for gluconeogenesis and glycolysis, respectively) + flux 53 (value of 1.1472 and 0.15 for gluconeogenesis and glycolysis, respectively) + flux 61 (value of -0.3789 and $-\mathbf{0 . 3 8}$ for gluconeogenesis and glycolysis, respectively). The Pareto curve M2 (shown as blue squares) is obtained after adding to M1 measured + flux 79 (value of 1.8962 and 1.9 for gluconeogenesis and glycolysis, respectively) + flux 81 (value of 0.0319 and 0.032 for gluconeogenesis and glycolysis, respectively). The Pareto curve M3 (shown as yellow triangles) is obtained after adding to M2 measured flux 66 (value of 0.0316 and 0.032 for gluconeogenesis and glycolysis, respectively) + flux 67 (value of -0.2292 and -0.23 for gluconeogenesis and glycolysis, respectively) + flux 68 (value of 0.1368 and 0.14 for gluconeogenesis and glycolysis, respectively). The Pareto curve M4 (shown as green stars) is obtained after adding to M3 measured flux 75 (value of 0.0978 and 0.098 for gluconeogenesis and glycolysis, respectively). Experimental data for gluconeogenesis and glycolysis were taken from (14) and (16), respectively.

When considering the ATP vs. albumin case (Fig. 9b), incorporation of measurements also changed the Pareto curve. At high- albumin secretion, the associated flux differences (Fig. 10c) included a significant increase in gluconeogenic fluxes (2-4), TCA cycle flux (8), and GSH (48) synthesis, a moderate increase in $\beta$-oxidation (40), a decrease in urea cycle fluxes $(14,15)$, urea secretion (16), lipid uptake (52) and albumin (47), a moderate decrease in electron transport (44) and NADPH (46) synthesis. At the anchor point of ATP generation on the Pareto curve, flux changes caused by introduction of the measurements (Fig. 10d) significantly increased gluconeogenesis fluxes (2-6), urea cycle fluxes $(14,15)$, and GSH (48) synthesis, moderately increased TCA cycle flux (8), urea secretion (16), and $\beta$-oxidation (40), decreased electron transport (44), lipid uptake (52), albumin synthesis (47), and NADPH (46). Additionally, there was decreased uptake of both gluconeogenic and ketogenic amino acids.
The effect of measurements on the Pareto curve of glutathione vs. urea are shown in Fig. 9c. The major differences in fluxes at the anchor point of high-urea secretion (Fig. 10e) included a significant increase in albumin (47), a moderate increase in TCA cycle flux (8), electron transport $(43,44)$, lipid uptake (52), and NADPH (46), a decrease in urea secretion (16), a moderate decrease in gluconeogenic fluxes (2-6), $\beta$-oxidation (40), and glutathione synthesis (48). As seen earlier, the increased albumin synthesis necessitates significant increase in the uptake of both gluconeogenic and ketogenic amino acids. The major differences in fluxes at the anchor point of high-glutathione synthesis (Fig. 10f) included a moderate increase in TCA cycle flux (8) and lipid storage, a significant increase in urea cycle fluxes $(14,15)$, a decrease in electron transport $(43,44)$, a moderate decrease in urea secretion (16) and lipid uptake (52), a significant decrease in $\beta$-oxidation (40) and glutathione synthesis (48). 
Urea-Albumin (Gluconeogenesis) System
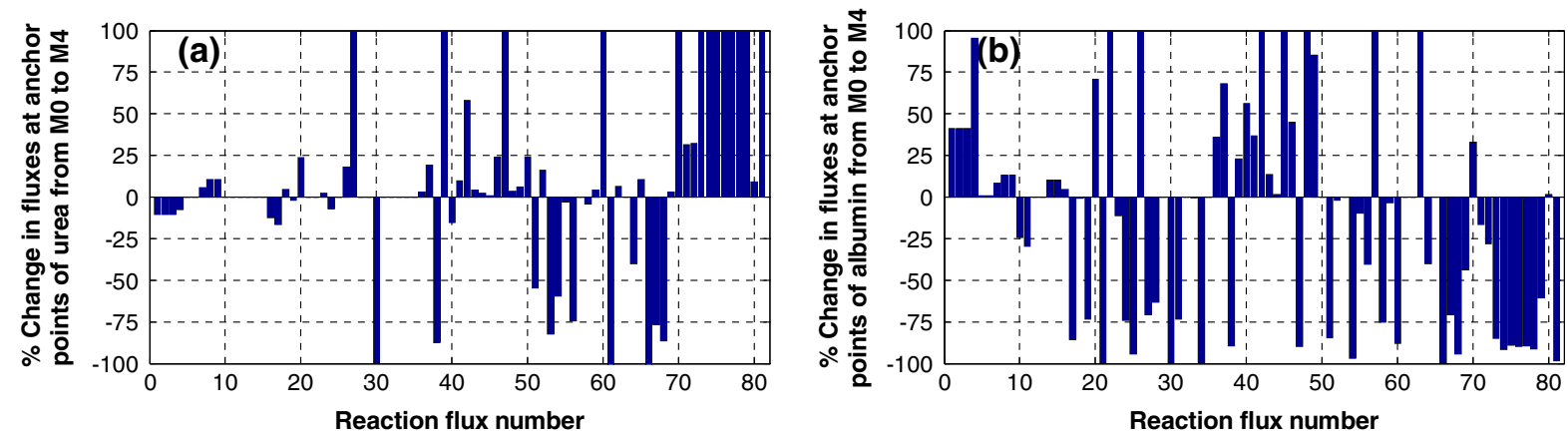

Albumin-ATP (Gluconeogenesis) System
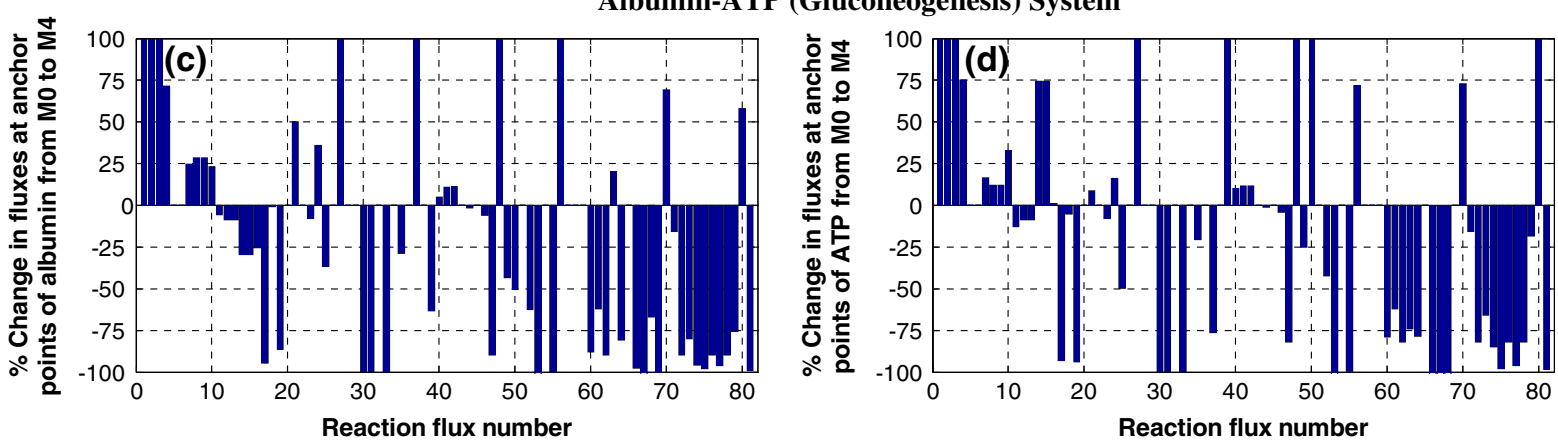

Urea-Glutathione (Gluconeogenesis) System
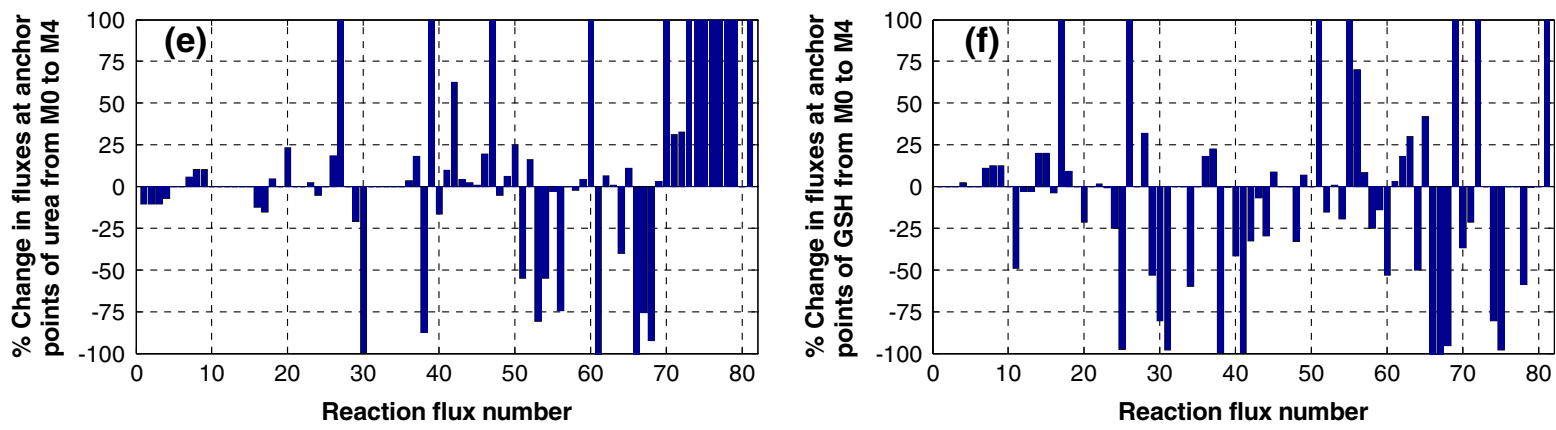

Urea-ATP (Glycolysis) System
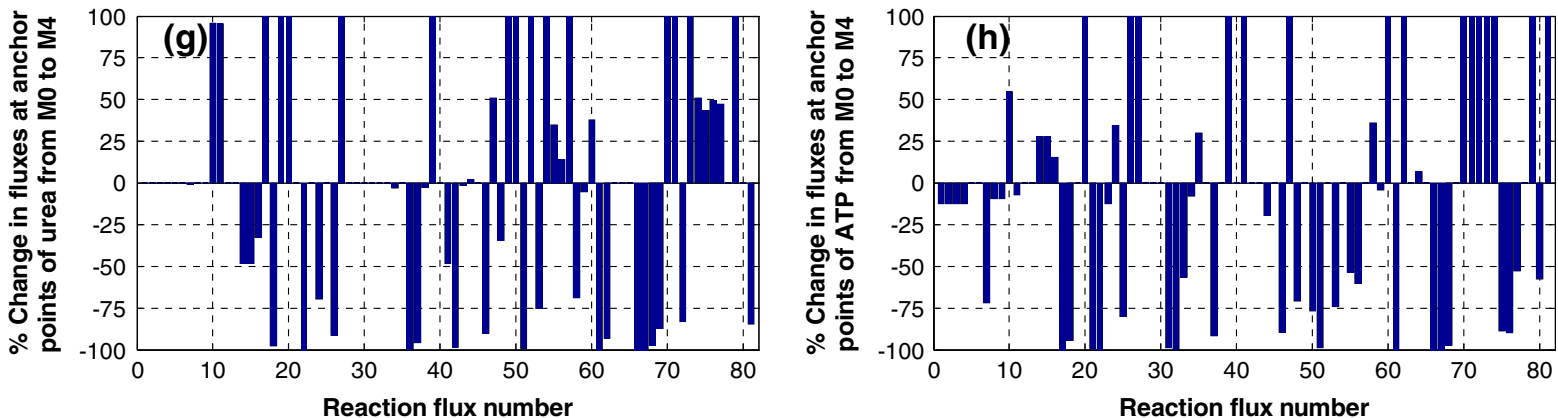

FIGURE 10. Distribution of optimal flux changes between the anchor points of the system solved without constraints (M0) and with the maximum number of constraints (M4) for the bi-objective system of Fig. 9. (a) and (b): albumin vs. urea (gluconeogenesis mode); (c) and (d): albumin vs. ATP (gluconeogenesis mode); (e) and (f): urea vs. glutathione (gluconeogenesis mode); (g) and (h): urea vs. ATP (glycolysis mode). The absolute flux values are in Table $\mathbf{S} 6$. Note that the $\%$ flux changes for all figures are on $y$-axis and the corresponding reaction flux number is shown on the horizontal axis. Only changes up to $100 \%$ are shown in the figure. 
TABLE 7. Objective function flux values for Pareto-optimal solutions in Fig. 4 (for gluconeogenic mode) and Fig. 6 (for glycolysis mode). The detailed flux values are provided in Tables S3 and S4.

\begin{tabular}{lcccccccccccc}
\hline Objectives & $\mathrm{A}$ & $\mathrm{B}$ & $\mathrm{C}$ & $\mathrm{D}$ & $\mathrm{E}$ & $\mathrm{F}$ & $\mathrm{G}$ & $\mathrm{H}$ & $\mathrm{I}$ & $\mathrm{J}$ & $\mathrm{K}$ & $\mathrm{L}$ \\
\hline Gluconeogenesis & & & & & & & & & & & & \\
Urea & 29.7685 & 34.56 & 10.431 & 10.595 & 19.752 & 12.454 & 30.13 & 34.465 & 2.8071 & 2.601 & 11.516 & 33.769 \\
NADPH & 0.47 & 0.53 & 1.414 & 0.98 & 2.985 & 2.786 & 1.091 & 0.825 & 1.134 & 1.411 & 1.008 & 0.945 \\
Albumin & 0.136 & 0.0126 & 0.011 & 0.1296 & 0.016 & 0.135 & 0.00001 & 0.00001 & 0.0992 & 0.139 & 0.0832 & 0.01136 \\
GSH & 6.837 & 8.79 & 14.427 & 9.585 & 14.4212 & 9.345 & 14.647 & 10.312 & 0.083 & 0.001 & 1.483 & 9.395 \\
Glycolysis & & & & & & & & & & & & \\
Urea & 26.717 & 29.365 & 14.441 & 14.388 & 12.323 & 9.978 & 25.304 & 29.368 & 8.695 & 6.28 & 12.264 & 28.234 \\
NADPH & 1.066 & 1.098 & 2.063 & 1.702 & 7.443 & 7.126 & 2.16 & 2.077 & 0.654 & 0.472 & 0.456 & 0.51 \\
Albumin & 0.131 & 0.02 & 0.012 & 0.1304 & 0.0131 & 0.137 & 0.00001 & 0.004 & 0.0232 & 0.133 & 0.0017 & 0.00001 \\
GSH & 5.226 & 9.175 & 14.404 & 9.549 & 14.36 & 9.276 & 14.473 & 10.173 & 6.614 & 1.296 & 7.007 & 9.909 \\
\hline
\end{tabular}

The effect of measurements on the Pareto curve of urea secretion vs. ATP generation (in glycolysis mode) are shown in Fig. 9d. The major differences in fluxes at the anchor points of high-urea secretion and ATP generations are shown in Fig. $10 \mathrm{~g}$ and $10 \mathrm{~h}$, respectively. We found that addition of the measurements did not change glycolysis fluxes (1-5) at either anchor point. On the other hand, at the anchor point of highurea secretion (Fig. 10g), there was a significant decrease in urea cycle fluxes $(14,15)$, urea secretion (16), NADPH (46) and GSH synthesis (48), a moderate decrease in electron transport (43), a significant increase in lipid uptake (52), lipid storage (57), and albumin (47). The increased albumin synthesis necessitates the increased uptake of gluconeogenic amino acids. The major differences in fluxes at the anchor point of highATP generation (Fig. 10h) included a moderate decrease in TCA cycle flux (8), a significant increase in urea cycle fluxes $(14,15)$ and albumin synthesis $(47)$, a moderate increase in urea secretion (16), a decrease in electron transport (44), and a significant decrease in NADPH and GSH synthesis. Additionally, there was a significant increase in the uptake of both gluconeogenic and ketogenic amino acids.

\section{DISCUSSION}

Mammalian cells exhibit various phenotypic states including proliferation, differentiation, etc. Metabolic flux distributions in these various states must obey constraints imposed by the environment, reaction stoichiometry, thermodynamics, and laws of conservation. Mathematically these constraints translate into a reduction of the feasible space for the flux distribution. Most of the literature on constraints-based metabolic network optimality deals with unicellular organisms where the main objective is growth of biomass. ${ }^{9,10}$ In mammalian systems, various phenotypes are encountered, some of which exhibit proliferation, and others expression of organ-specific or "differentiated" functions. Several objectives should be considered before making any conclusions about the optimal states of such systems. Often times there is a competition between the various objectives because they are differentially altered by the constraints. This paradigm of conflicting objectives is addressed herein using a class of multi-objective optimality called Pareto optimality. Furthermore, we used the Normal Constraint method, which yields any Pareto point in the feasible objective space, guarantees an even distribution of the Pareto frontier, and is insensitive to design objective scaling. Combining these concepts with FBA and EBA, we developed a framework called NCEFBA, which we applied to the specific case of cultured hepatocytes.

Hepatocytes are the key cellular component in BAL devices. The ability to optimize hepatocellular metabolism is important to maximize the clinical efficacy of the BAL, and increasing the function per cell may help reduce the number of hepatocytes needed in the device. Hepatocytes express various liver-specific functions that require common substrates, such as glucose, amino acids, and so on. Thus, it is expected that increasing one function (for instance, albumin synthesis) will decrease another (for example, urea secretion). In order to systematically investigate the tradeoffs between the various hepatocellular functions, we used NCEFBA. More specifically, we investigated the interactions among five key hepatocyte metabolic functions, namely albumin synthesis, urea secretion, glutathione synthesis, NADPH synthesis, and ATP generation. These analyses were carried out first in gluconeogenic hepatocytes (Fig. 4) and then glycolytic hepatocytes (Fig. 6).

Using NCEFBA, we observed the Pareto optimality between various liver-specific functions. Some of the representative bi-objective combinations were shown in this paper. Here, the implementation was done for several biobjective combinations in order to develop a 
suitable framework for designing a compartmental BAL device that can perform all essential liver-specific functions. This BAL device could have several individual bioreactor modules interconnected in series and each individual bioreactor could be designed based on the various combinations of liver-specific Pareto-optimal solutions. The idea here will be to obtain an optimal BAL system that can exhibit very high and stable levels of key liver-specific functions and thus translate into a proportional reduction of required cell mass and total perfusion volume of the bioreactor required for a given processing capacity. The six combinations of liver-specific functions for both gluconeogenic hepatocytes and glycolytic hepatocytes were analyzed to obtain a global Pareto-optimal solution with respect to each liver-specific function in BAL assembly. Table 7 shows the flux values for these solutions at few representative Pareto-optimal solutions $\mathrm{A}, \mathrm{B}, \mathrm{C}, \mathrm{D}, \mathrm{E}$, and $\mathrm{F}$ for both gluconeogenic and glycolytic hepatocytes of Figs. 4 and 6. Based on the results obtained, to design a BAL assembly system that provides higher liver-specific functions in gluconeogenic mode, one option could be to operate five different bioreactors in series at $\mathrm{H}, \mathrm{G}, \mathrm{F}, \mathrm{E}$, and $\mathrm{D}$ points, respectively from Fig. 4. If the five reactors are operated at these points then the total fluxes can be calculated by summing up the individual fluxes at these points. For albumin, urea, glutathione, and NADPH synthesis these values are $0.281,107.4,58.31$, and 8.67, respectively. On the other hand, if the reactor assembly is just operated at the equal preference point of $E$ then the total fluxes of albumin, urea, glutathione and NADPH synthesis will be $0.08,98.76,72.1$, and 14.93 , respectively. This indicates that the variable operating condition BAL will produce overall higher albumin and urea synthesis compared to the case where assembly is just operated at point $\mathrm{E}$ condition. It is to be noted that glutathione and NADPH synthesis in variable operating condition reactor is lower than that if assembly is operated at point $\mathrm{E}$ alone. This could be tolerable because of higher priority to attain highalbumin and -urea synthesis. However, if there is a situation where there is a higher demand of ATP (because of stress and mitochondrial dysfunction) BAL system for gluconeogenic mode could be designed for $\mathrm{H}, \mathrm{G}, \mathrm{J}, \mathrm{K}$, and L points, resepectively from Fig. 4. In glycolytic mode of BAL operation the preferred combination of reactor operations could be $\mathrm{H}, \mathrm{G}, \mathrm{C}, \mathrm{D}$, and $\mathrm{F}$ points, resepectively from Fig. 6 . If the five bioreactors are operated at these points then the total fluxes of albumin, urea, glutathione, and NADPH synthesis are $0.283,93.48,57.88$, and 15.13 , respectively. On the other hand, if the reactor assembly is just operated at the equal preference point of $\mathrm{C}$ then the total fluxes of albumin, urea, glutathione, and
NADPH synthesis are $0.06,72.21,72.02$, and 10.32, respectively. As seen earlier for gluconeogenic hepatocytes, we see also in glycolytic hepatocytes that the variable operating condition BAL will produce in overall higher albumin, urea, and NADPH synthesis compared to the case where assembly is operated at point $\mathrm{C}$ condition. Again, if there is a situation demanding higher energy production BAL system for glycolytic mode could be H, G, I, K, and L point, respectively from Fig. 6 .

The NCEFBA platform is a useful tool for optimality analysis of large-scale metabolic networks that are bound to possess multi-objective Pareto-optimal solutions. This technique enables the systematic identification of tradeoff situations between various metabolic objectives that characterize a particular cellular phenotype. The addition of FBA to EBA constraints ensures that thermodynamically feasible solutions are obtained. Furthermore, experimental flux data can be easily incorporated into the analysis, which further reduces the feasible space of fluxes. Although the NCEFBA approach described here was applied to the specific case of hepatocellular metabolism, it can be readily used on any large-scale metabolic network. It is noteworthy that as part of the future work, the optimal fluxes obtained through multi-bojective optimization are currently being experimentally investigated by using hormonal supplements, inducers, and transfection of primary heptocytes in our laboratory. Importantly, since the presented hepatic metabolic network model has reasonable predictability for both whole organ (liver) and in vitro hepatic systems it can be readily applied to BAL systems.

In conclusion, this study highlights how Paretooptimal solutions may contribute to operating BAL devices, alter the metabolic states of hepatocytes, achieve the desired range of objectives and has relevance for understanding the impact of environmental stress, inducers, hormones, and supplements on cellular metabolism. The important contribution of the paper is that it presents a strategy for the coupling of Normalized Constraint multi-objective method with EBA to obtain "true optimal solutions" throughout the feasible space. The simple method like "weighted sum" fails to capture the points that are in the concave part of the Pareto frontier. However, using the presented approach it is possible to capture every Pareto point given the generic morphology of an objective function.

\section{ELECTRONIC SUPPLEMENTARY MATERIAL}

The online version of this article (doi:10.1007/ s10439-007-9283-0) contains supplementary material, which is available to authorized users. 


\section{ACKNOWLEDGMENTS}

The authors thank Don Poulsen for his assistance in designing Fig. 1. This work was funded by grants from the NIH (DK 043371 and DK 059766) and from the Shriners Hospital for Children.

\section{REFERENCES}

${ }^{1}$ Banta, S., T. Yokoyama, F. Berthiaume, and M. L. Yarmush. Quantitative effects of thermal injury and insulin on the metabolism of the skeletal muscle using the perfused rat hindquarter preparation. Biotechnol. Bioeng. 88:613-629, 2004.

${ }^{2}$ Beard, D. A., E. Babson, E. Curtis, and H. Qian. Thermodynamic constraints for biochemical networks. J. Theor. Biol. 228:327-333, 2004.

${ }^{3}$ Beard, D. A., S. D. Liang, and H. Qian. Energy balance for analysis of complex metabolic networks. Biophys. J. 83:79-86, 2002.

${ }^{4}$ Beard, D. A., and H. Qian. Thermodynamic-based computational profiling of cellular regulatory control in hepatocyte metabolism. Am. J. Physiol. Endocrinol. Metab. 288:E633-E644, 2005.

${ }^{5}$ Chan, C., F. Berthiaume, K. Lee, and M. L. Yarmush. Metabolic flux analysis of cultured hepatocytes exposed to plasma. Biotechnol. Bioeng. 81:33-49, 2003.

${ }^{6}$ Chan, C., F. Berthiaume, K. Lee, and M. L. Yarmush. Metabolic flux analysis of hepatocyte function in hormoneand amino acid-supplemented plasma. Metab. Eng. 5:1-15, 2003.

${ }^{7}$ Chan, C., F. Berthiaume, J. Washizu, M. Toner, and M. L. Yarmush. Metabolic pre-conditioning of cultured cells in physiological levels of insulin: generating resistance to the lipid-accumulating effects of plasma in hepatocytes. Biotechnol. Bioeng. 78:753-760, 2002.

${ }^{8}$ Chan, C., D. Hwang, G. N. Stephanopoulos, M. L. Yarmush, and G. Stephanopoulos. Application of multivariate analysis to optimize function of cultured hepatocytes. Biotechnol. Prog. 19:580-598, 2003.

${ }^{9}$ Edwards, J. S., and B. O. Palsson. Metabolic flux balance analysis and the in silico analysis of Escherichia coli K-12 gene deletions. BMC Bioinform. 1:1, 2000.

${ }^{10}$ Edwards, J. S., and B. O. Palsson. Robustness analysis of the Escherichia coli metabolic network. Biotechnol. Prog. 16:927-939, 2000.

${ }^{11}$ Lee, K., F. Berthiaume, G. N. Stephanopoulos, D. M. Yarmush, and M. L. Yarmush. Metabolic flux analysis of postburn hepatic hypermetabolism. Metab. Eng. 2:312$327,2000$.
${ }^{12}$ Lee, K., F. Berthiaume, G. N. Stephanopoulos, and M. L. Yarmush. Profiling of dynamic changes in hypermetabolic livers. Biotechnol. Bioeng. 83:400-415, 2003.

${ }^{13}$ Lee, K., F. Berthiaume, G. N. Stephanopoulos, and M. L. Yarmush. Induction of a hypermetabolic state in cultured hepatocytes by glucagon and $\mathrm{H}_{2} \mathrm{O}_{2}$. Metab. Eng. 5:221229, 2003.

${ }^{14}$ Lee, K., D. Hwang, T. Yokoyama, G. Stephanopoulos, G. N. Stephanopoulos, and M. L. Yarmush. Identification of optimal classification functions for biological sample and state discrimination from metabolic profiling data. Bioinformatics 20:959-969, 2004.

${ }^{15}$ Messac, A., A. Ismail-Yahaya, and C. A. Mattson. The normalized normal constraint method for generating the Pareto frontier. Struct. Multidiscipl. Optimiz. 25:86-98, 2003.

${ }^{16}$ Nagrath, D., A. Messac, B. W. Bequette, and S. M. Cramer. Multiobjective optimization strategies for linear gradient chromatography. AIChE J. 51:511-525, 2005.

${ }^{17}$ Papin, J. A., N. D. Price, J. S. Edwards, and B. B. Palsson. The genome-scale metabolic extreme pathway structure in Haemophilus influenzae shows significant network redundancy. J. Theor. Biol. 215:67-82, 2002.

${ }^{18}$ Savinell, J. M., and B. O. Palsson. Network analysis of intermediary metabolism using linear optimization. I. Development of mathematical formalism. J. Theor. Biol. 154:421-454, 1992.

${ }^{19}$ Schilling, C. H., M. W. Covert, I. Famili, G. M. Church, J. S. Edwards, and B. O. Palsson. Genome-scale metabolic model of Helicobacter pylori 26695. J. Bacteriol. 184:45824593, 2002.

${ }^{20}$ Segre, D., A. Deluna, G. M. Church, and R. Kishony. Modular epistasis in yeast metabolism. Nat. Genet. 37:7783, 2005.

${ }^{21}$ Segre, D., D. Vitkup, and G. M. Church. Analysis of optimality in natural and perturbed metabolic networks. Proc. Natl. Acad. Sci. USA 99:15112-15117, 2002.

${ }^{22}$ Vo, T. D., H. J. Greenberg, and B. O. Palsson. Reconstruction and functional characterization of the human mitochondrial metabolic network based on proteomic and biochemical data. J. Biol. Chem. 279:39532-39540, 2004.

${ }^{23}$ Yang, F., H. Qian, and D. A. Beard. Ab initio prediction of thermodynamically feasible reaction directions from biochemical network stoichiometry. Metab. Eng. 7:251259, 2005.

${ }^{24}$ Yarmush, M. L., and S. Banta. Metabolic engineering: advances in modeling and intervention in health and disease. Annu. Rev. Biomed. Eng. 5:349-381, 2003.

${ }^{25}$ Yokoyama, T., S. Banta, F. Berthiaume, D. Nagrath, R. G. Tompkins, and M. L. Yarmush. Evolution of intrahepatic carbon, nitrogen, and energy metabolism in a D-galactosamine-induced rat liver failure model. Metab. Eng. 7:88$103,2005$. 